\title{
A Versatile Route to L-Hexoses: Synthesis of L- Mannose and L-Altrose
}

\author{
Annalisa Guaragna,* Carmela Napolitano, Daniele D’Alonzo, Silvana Pedatella and Giovanni Palumbo \\ Dipartimento di Chimica Organica e Biochimica, Università di Napoli Federico II \\ via Cynthia, 4 I-80126 Napoli, Italy \\ guaragna@unina.it
}

\section{CONTENTS}

EXPERIMENTAL PROCEDURES

${ }^{1} \mathrm{H}$ NMR SPECTRA

Compound anti-5

S14

Compound syn-5

S15

Compound 6

S16

Compound 7

S17

Compound 8

S18

Compound 9

S19

Compound 10

S20

Compound 12

S21

Compound 13

S22

Compound 14

S23

Compound 15

S24

Compound 16

S25

Compound 17

S26

Compound 18

S27

Compound 20

S28

Compound 21

S29

Compound 22

S30

${ }^{13}$ C NMR SPECTRA

Compound 6

Compound 7

S32

Compound 9

S33

Compound 13

S34

Compound 14

S35

Compound 16

S36 


\section{D NMR SPECTRA}

All moisture-sensitive reactions were performed under a nitrogen atmosphere using oven-dried glassware. Solvents were dried over standard drying agents and freshly distilled prior to use. Reactions were monitored by TLC (precoated silica gel plate $\mathrm{F}_{254}$, Merck). Column chromatography: Merck Kieselgel 60 (70-230 mesh); flash chromatography: Merck Kieselgel 60 (230-400 mesh). Melting points are uncorrected and were determined with a capillary apparatus. Optical rotations were measured at 25 $\pm 2{ }^{\circ} \mathrm{C}$ in the stated solvent. ${ }^{1} \mathrm{H}$ and ${ }^{13} \mathrm{C}$ NMR spectra were recorded on NMR spectrometers operating at 200, 300, 400 or $500 \mathrm{MHz}$ and 50, 75, 100 or $125 \mathrm{MHz}$, respectively. Wherever necessary, twodimensional H-H COSY experiments were carried out for complete signal assignments. Combustion analyses were performed using CHNS analyzer.

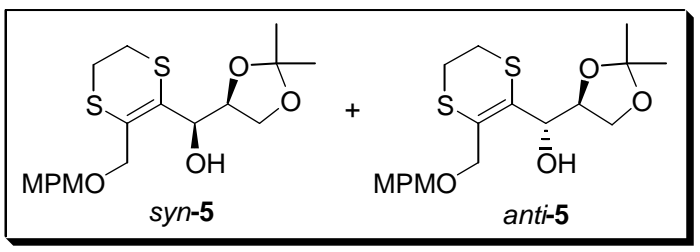

Compounds syn-5 and anti-5. BuLi (1.6 M in hexane, $2.82 \mathrm{~mL})$ was added dropwise to a stirred solution of 1 (1.01 g, $3.76 \mathrm{mmol})$ in anhydrous THF $(5 \mathrm{~mL})$ at $-78^{\circ} \mathrm{C}$ and under nitrogen atmosphere. After $10 \mathrm{~min}$ a solution of $(S)$-glyceraldehyde acetonide $(0.64 \mathrm{~g}, 4.89 \mathrm{mmol})$ in the same solvent $(3 \mathrm{~mL})$ was added. The reaction mixture was stirred for $30 \mathrm{~min}$ at $-78^{\circ} \mathrm{C}$, then carefully quenched with $10 \%$ aq $\mathrm{NH}_{4} \mathrm{Cl}$. The mixture was extracted with EtOAc, the combined organic phases washed with brine, dried $\left(\mathrm{Na}_{2} \mathrm{SO}_{4}\right)$ and evaporated under reduced pressure. Flash chromatography of the crude residue over silica 
gel (hexane/Et $\mathrm{t}_{2} \mathrm{O}=7: 3$ ) gave the two separated diastereomers syn-5 and anti-5 (1.42 g, 95\% overall yield, 4:6 dr).

Lower $R_{f}$ compound syn-5 (0.86 g, 57\% yield): oily, $[\alpha]_{\mathrm{D}}^{25}-16.6\left(c\right.$ 0.64, $\left.\mathrm{CHCl}_{3}\right) .{ }^{1} \mathrm{H} \mathrm{NMR}(500 \mathrm{MHz}$, $\left.\mathrm{CDCl}_{3}\right): \delta 1.38(\mathrm{~s}, 3 \mathrm{H}), 1.44$ (s, 3H), 1.60 (bs, $\left.1 \mathrm{H}\right), 3.10-3.15(\mathrm{~m}, 2 \mathrm{H}), 3.18-3.28(\mathrm{~m}, 2 \mathrm{H}), 3.78(\mathrm{dd}, J=$ 8.6, $J=5.9 \mathrm{~Hz}, 1 \mathrm{H}$ ), 3.80 (s, 3H), 3.97 (dd, $J=8.6, J=6.5 \mathrm{~Hz}, 1 \mathrm{H}), 4.00$ (d, $J=12.0 \mathrm{~Hz}, 1 \mathrm{H}), 4.15$ (d, $J=12.0 \mathrm{~Hz}, 1 \mathrm{H}), 4.33$ (ddd, $J=7.4, J=6.5, J=5.9 \mathrm{~Hz}, 1 \mathrm{H}), 4.46$ (d, $J=11.4 \mathrm{~Hz}, 1 \mathrm{H}), 4.50$ (d, $J=$

$11.4 \mathrm{~Hz}, 1 \mathrm{H}), 4.56$ (d, $J=7.4 \mathrm{~Hz}, 1 \mathrm{H}), 6.78$ (d, $J=8.6 \mathrm{~Hz}, 2 \mathrm{H}), 7.28$ (d, $J=8.6 \mathrm{~Hz}, 2 \mathrm{H}) .{ }^{13} \mathrm{C}$ NMR $(100$ $\left.\mathrm{MHz}, \mathrm{CDCl}_{3}\right): \delta$ 25.2, 26.6, 27.3, 29.7, 55.3, 66.6, 70.2, 71.6, 73.1, 76.5, 109.3, 113.9, 125.9, 129.4, 129.7, 130.2, 159.7. Anal. calcd for $\mathrm{C}_{19} \mathrm{H}_{26} \mathrm{O}_{5} \mathrm{~S}_{2}$ : C, 57.26; H, 6.58. Found: C, 57.40; H, 6.61.

Higher $R_{f}$ compound anti-5 (0.56 g, 38\% yield): oily, $[\alpha]_{\mathrm{D}}^{25}-32.9$ (c 0.49, $\left.\mathrm{CHCl}_{3}\right) .{ }^{1} \mathrm{H}$ NMR (500 MHz, $\left.\mathrm{CDCl}_{3}\right): \delta 1.35(\mathrm{~s}, 3 \mathrm{H}), 1.38(\mathrm{~s}, 3 \mathrm{H}), 3.06-3.18(\mathrm{~m}, 2 \mathrm{H}), 3.20-3.23(\mathrm{~m}, 2 \mathrm{H}), 3.80(\mathrm{~s}, 3 \mathrm{H}), 3.90(\mathrm{~d}, J=$ $11.7 \mathrm{~Hz}, 1 \mathrm{H}$ ), 4.03 (dd, $J=8.8, J=5.9 \mathrm{~Hz}, 1 \mathrm{H}), 4.06$ (dd, $J=8.8, J=5.8 \mathrm{~Hz}, 1 \mathrm{H}), 4.22$ (ddd, $J=6.8, J$ $=5.9, J=5.8 \mathrm{~Hz}, 1 \mathrm{H}), 4.29(\mathrm{~d}, J=11.7 \mathrm{~Hz}, 1 \mathrm{H}), 4.45(\mathrm{~d}, J=11.7 \mathrm{~Hz}, 1 \mathrm{H}), 4.50(\mathrm{~d}, J=11.7 \mathrm{~Hz}, 1 \mathrm{H})$, $4.66(\mathrm{~d}, J=6.8 \mathrm{~Hz}, 1 \mathrm{H}), 6.87$ (d, $J=8.6 \mathrm{~Hz}, 2 \mathrm{H}), 7.28$ (d, $J=8.6 \mathrm{~Hz}, 2 \mathrm{H}) .{ }^{13} \mathrm{C}$ NMR $(125 \mathrm{MHz}$, $\left.\mathrm{CDCl}_{3}\right): \delta 25.2,26.6,27.3,29.8,55.3,66.6,70.2,71.6,72.1,76.6,109.3,113.9,126.0,129.5,129.7$, 130.1, 159.5. Anal. calcd for $\mathrm{C}_{19} \mathrm{H}_{26} \mathrm{O}_{5} \mathrm{~S}_{2}$ : C, 57.26; H, 6.58. Found: C, 57.42; H, 6.56.

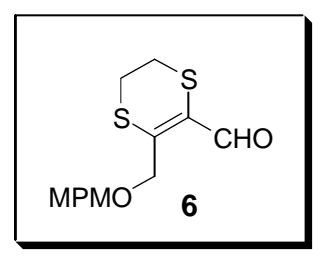

Compound 6. Under similar conditions reported above, treatment of 1 (1.01 g, 3.76 mmol) with BuLi (1.6 $\mathrm{M}$ in hexane, $2.82 \mathrm{~mL})$ and $2(0.64 \mathrm{~g}, 4.89 \mathrm{mmol})$ in the presence of a $20 \%$ amount of $\mathrm{Ti}(\mathrm{O}-\mathrm{i}-\mathrm{Pr})_{4}$ led, after usual work-up and purification procedures, to the pure oil 6 (0.96 g, $86 \%$ yield), besides traces of alcohols 5. ${ }^{1} \mathrm{H}$ NMR (500 MHz, $\left.\mathrm{CDCl}_{3}\right): \delta 3.10-3.18(\mathrm{~m}, 2 \mathrm{H}), 3.28-3.32(\mathrm{~m}, 2 \mathrm{H}), 3.81$ (s, 3H), 4.41 (s, 2H), 4.52 (s, 2H), 6.89 (d, $J=8.3 \mathrm{~Hz}, 2 \mathrm{H}), 7.26$ (d, $J=8.3 \mathrm{~Hz}, 2 \mathrm{H}), 9.82(\mathrm{~s}, 1 \mathrm{H}) .{ }^{13} \mathrm{C}$ NMR 
(125 MHz, $\left.\mathrm{CDCl}_{3}\right): \delta$ 25.5, 30.2, 55.3, 68.9, 72.2, 114.0, 129.0, 129.6, 148.8, 159.6, 183.5. Anal.calcd for $\mathrm{C}_{14} \mathrm{H}_{16} \mathrm{O}_{3} \mathrm{~S}_{2}$ : C, 56.73; H, 5.44. Found: C, 56.90; H, 5.42.

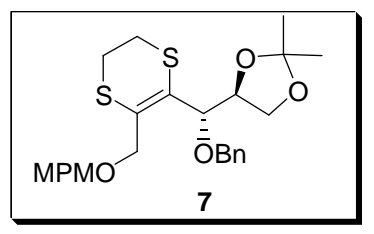

Compound 7. $\mathrm{NaH}(0.07 \mathrm{~g}, 1.83 \mathrm{mmol})$ was added to a solution of anti-5 (0.56 g, $1.41 \mathrm{mmol})$ in anhydrous DMF $(12 \mathrm{~mL})$ at $0{ }^{\circ} \mathrm{C}$ under nitrogen atmosphere. After $10 \mathrm{~min}$ benzyl bromide $(0.23 \mathrm{~mL}$, $1.93 \mathrm{mmol}$ ) was added in one portion. The reaction mixture was warmed to room temperature, stirred for $2 \mathrm{~h}$, then carefully quenched with $10 \%$ aq $\mathrm{NH}_{4} \mathrm{Cl}$. The mixture was extracted with EtOAc, the combined organic phases washed with brine, dried $\left(\mathrm{Na}_{2} \mathrm{SO}_{4}\right)$ and evaporated under reduced pressure. Chromatography of the crude residue over silica gel (hexane/ $\mathrm{Et}_{2} \mathrm{O}=8: 2$ ) afforded the pure 7 (0.66 g, 95\% yield): oily, $[\alpha]_{\mathrm{D}}^{25}-26.1$ (c 0.54, $\left.\mathrm{CHCl}_{3}\right) .{ }^{1} \mathrm{H}$ NMR (500 MHz, $\left.\mathrm{CDCl}_{3}\right): \delta 1.31$ (s, 6H), 3.08-3.14 (m, 1H), 3.17-3.23 (m, 2H), 3.27-3.34 (m, 1H), 3.78 (d, $J=11.7 \mathrm{~Hz}, 1 \mathrm{H}), 3.80$ (s, 3H), 3.86 (dd, $J=8.8, J=$ $5.8 \mathrm{~Hz}, 1 \mathrm{H}), 4.09$ (dd, $J=8.8, J=6.8 \mathrm{~Hz}, 1 \mathrm{H}), 4.24-4.30$ (m, 1H), 4.31 (d, $J=11.6 \mathrm{~Hz}, 1 \mathrm{H}), 4.40$ (d, $J$ $=7.8 \mathrm{~Hz}, 1 \mathrm{H}), 4.41(\mathrm{~d}, J=11.7 \mathrm{~Hz}, 1 \mathrm{H}), 4.44(\mathrm{~d}, J=11.7 \mathrm{~Hz}, 1 \mathrm{H}), 4.48(\mathrm{~d}, J=11.7 \mathrm{~Hz}, 1 \mathrm{H}), 4.64(\mathrm{~d}, J$ $=11.7 \mathrm{~Hz}, 1 \mathrm{H}), 6.86(\mathrm{~d}, J=8.6 \mathrm{~Hz}, 2 \mathrm{H}), 7.24-7.33(\mathrm{~m}, 7 \mathrm{H}) .{ }^{13} \mathrm{C} \mathrm{NMR}\left(125 \mathrm{MHz}, \mathrm{CDCl}_{3}\right): \delta$ 25.2, 25.9, 27.4, 29.7, 55.4, 67.5, 69.8, 70.5, 72.1, 76.2, 79.2, 109.3, 114.0, 127.3, 127.9, 128.1, 128.3, 129.7, 130.0, 137.9, 159.2. Anal. calcd for $\mathrm{C}_{26} \mathrm{H}_{32} \mathrm{O}_{5} \mathrm{~S}_{2}$ : C, 63.90; H, 6.60. Found: C, 64.12; H, 6.57. 


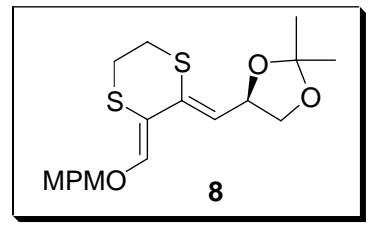

Compound 8. Under similar conditions reported above, treatment of anti-5 ( $0.30 \mathrm{~g}, 0.75 \mathrm{mmol})$ with an excess of $\mathrm{NaH}(1.5 \mathrm{mmol})$ afforded, after common work-up and purification procedures, the pure 7 (0.24 g, 65\% yield) besides a remarkable amount of 8 (0.06 g, 20\% yield): oily, $[\alpha]_{\mathrm{D}}^{25}-61.8$ (c 0.43 , $\mathrm{CHCl}_{3}$ ). ${ }^{1} \mathrm{H}$ NMR (500 MHz, $\mathrm{CDCl}_{3}$ ): $\delta 1.38$ (s, 3H), 1.41 (s, 3H), 3.00-3.15 (m, 4H), 3.54 (t, $J=6.8$ Hz, 1H), 3.81 (s, 3H), 4.11 (t, $J=6.8 \mathrm{~Hz}, 1 \mathrm{H}), 4.86-4.94$ (m, 3H), 5.56 (d, $J=8.3 \mathrm{~Hz}, 1 \mathrm{H}), 6.64$ (s, 1H), $6.90(\mathrm{~d}, J=8.3 \mathrm{~Hz}, 2 \mathrm{H}), 7.28$ (d, $J=8.3 \mathrm{~Hz}) .{ }^{13} \mathrm{C}$ NMR (125 MHz, $\left.\mathrm{CDCl}_{3}\right): \delta$ 25.8, 26.7, 29.7, 30.0, 55.3, 68.9, 73.1, 74.4, 108.7, 109.2, 114.0, 122.7, 128.6, 129.2, 135.7, 144.3, 159.7. Anal. calcd for $\mathrm{C}_{19} \mathrm{H}_{24} \mathrm{O}_{4} \mathrm{~S}_{2}$ : C, 59.97; H, 6.36. Found: C, 59.85; H, 6.34.

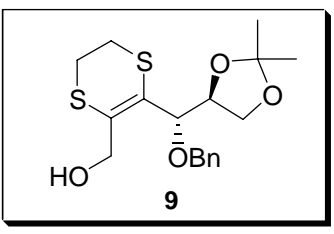

Compound 9. Method A: to a stirred $\mathrm{CH}_{2} \mathrm{Cl}_{2} / \mathrm{H}_{2} \mathrm{O}$ (18:1) emulsion (5 mL) containing the MPM ether 7 (0.54 g, $1.10 \mathrm{mmol})$, DDQ (0.38 g, $1.68 \mathrm{mmol}$ ) was added in one portion at room temperature. After 12 h $\mathrm{H}_{2} \mathrm{O}$ was added to the reaction and the mixture extracted with $\mathrm{CH}_{2} \mathrm{Cl}_{2}$; the organic layer was dried $\left(\mathrm{Na}_{2} \mathrm{SO}_{4}\right)$ and the solvent evaporated under reduced pressure. Chromatography of the crude residue over silica gel (hexane/acetone $=95: 5)$ gave the pure $\mathbf{9}(0.12 \mathrm{~g}, 29 \%$ yield) beside a remarkable amount of $\mathbf{1 0}$ (0.08 g, 19\% yield). Method B: to a stirred $\mathrm{CH}_{2} \mathrm{Cl}_{2} / \mathrm{H}_{2} \mathrm{O}$ (9:1) emulsion (50 mL) containing the MPM ether 7 (0.54 g, $1.10 \mathrm{mmol})$, DDQ (0.38 g, $1.68 \mathrm{mmol})$ was added in one portion at room temperature. After $3 \mathrm{~h} \mathrm{H}_{2} \mathrm{O}$ was added to the reaction and the mixture extracted with $\mathrm{CH}_{2} \mathrm{Cl}_{2}$; the organic layer was dried $\left(\mathrm{Na}_{2} \mathrm{SO}_{4}\right)$ and the solvent evaporated under reduced pressure. Chromatography of the crude residue over silica gel (hexane/acetone $=95: 5)$ gave the pure $9\left(0.28 \mathrm{~g} ; 70 \%\right.$ yield): oily, $[\alpha]_{\mathrm{D}}^{25}-61.8(c$ 
0.43, $\mathrm{CHCl}_{3}$ ). ${ }^{1} \mathrm{H}$ NMR (500 MHz, $\mathrm{CDCl}_{3}$ ): $\delta 1.36$ (s, 3H), 1.40 (s, 3H), 3.04-3.11 (m, 1H), 3.19-3.26 (m, 2H), 3.31-3.36 (m, 1H), 3.71 (d, $J=13.2 \mathrm{~Hz}, 1 \mathrm{H}), 3.76-3.79(\mathrm{~m}, 1 \mathrm{H}), 4.17-4.22(\mathrm{~m}, 1 \mathrm{H}), 4.28$ (d, $J$ $=11.7 \mathrm{~Hz}, 1 \mathrm{H}), 4.32-4.34(\mathrm{~m}, 2 \mathrm{H}), 4.36(\mathrm{~d}, J=13.2 \mathrm{~Hz}, 1 \mathrm{H}), 4.65$ (d, $J=11.7 \mathrm{~Hz}, 1 \mathrm{H}), 7.28-7.40$ (m, 5H). ${ }^{13} \mathrm{C}$ NMR (75 MHz, $\left.\mathrm{CDCl}_{3}\right): \delta 24.9,25.9,26.7,29.9,62.8,68.1,70.3,74.7,78.4,109.9,127.1$, 128.0, 128.2, 128.4, 131.2, 137.0. Anal. calcd for $\mathrm{C}_{18} \mathrm{H}_{24} \mathrm{O}_{4} \mathrm{~S}_{2:}$ C, 58.67; H, 6.56. Found: C, 58.55; $\mathrm{H}$ 6.58.

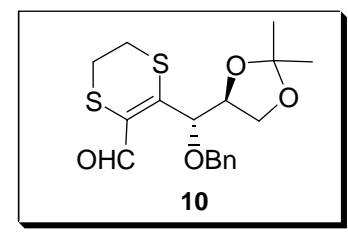

Compound 10. A solution of alcohol 9 (0.28 g, $0.75 \mathrm{mmol})$ in Py (2 mL) was added in one portion to a stirred suspension of PCC $(0.22 \mathrm{~g}, 1.02 \mathrm{mmol})$ and Celite $(0.22 \mathrm{~g})$ in Py $(7 \mathrm{~mL})$ at room temperature. The resulting mixture was stirred for $8 \mathrm{~h}$ and then diluted with $10 \mathrm{~mL}$ of anhydrous $\mathrm{Et}_{2} \mathrm{O}$, kept in ultrasound bath for $30 \mathrm{~min}$, and filtered on a pad of silica gel. After solvent removal under reduced pressure, chromatography of the crude residue over silica gel $\left(\mathrm{CH}_{2} \mathrm{Cl}_{2}\right)$ gave the pure $\mathbf{1 0}(0.27 \mathrm{~g}, 99 \%$ yield): white solid, mp 91.7-93.3 ${ }^{\circ} \mathrm{C}$ (from $\left.\mathrm{MeOH}\right),[\alpha]_{\mathrm{D}}^{25}+35.5\left(c \quad 0.53, \mathrm{C}_{6} \mathrm{H}_{6}\right) .{ }^{1} \mathrm{H}$ NMR (500 MHz, $\left.\mathrm{CDCl}_{3}\right): \delta 1.30$ (s, 3H), 1.34 (s, 3H), 3.15-3.22 (m, 2H), 3.23-3.38 (m, 2H), $3.92(\mathrm{dd}, J=8.8, J=4.8 \mathrm{~Hz}$, $1 \mathrm{H}), 4.18$ (dd, $J=8.8, J=6.8 \mathrm{~Hz}, 1 \mathrm{H}), 4.26$ (ddd, $J=7.8, J=6.8, J=4.8 \mathrm{~Hz}, 1 \mathrm{H}), 4.37$ (d, $J=11.3 \mathrm{~Hz}$, 1H), 4.71 (d, $J=11.3 \mathrm{~Hz}, 1 \mathrm{H}), 4.80$ (d, $J=7.8 \mathrm{~Hz}, 1 \mathrm{H}), 7.30-7.40$ (m, 5H), $9.84(\mathrm{~s}, 1 \mathrm{H}) .{ }^{13} \mathrm{C}$ NMR $(125$ $\left.\mathrm{MHz}, \mathrm{CDCl}_{3}\right): \delta 24.9,26.1,27.3,29.1,67.6,71.5,76.4,78.4,110.1,128.3,128.6,131.1,136.5,151.5$, 182.5. Anal. Calcd for $\mathrm{C}_{18} \mathrm{H}_{22} \mathrm{O}_{4} \mathrm{~S}_{2}$ : C, 58.99; H, 6.05. Found: C, 58.75; H 6.08. 


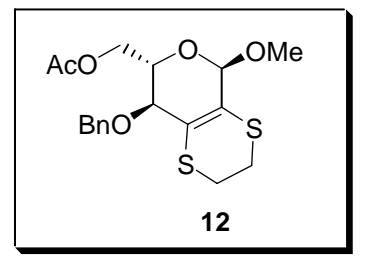

Compound 12. Amberlyst 15 (1.2 g, previously washed with anhydrous $\mathrm{MeOH}$ ), was added in one portion to a stirred solution of aldehyde $\mathbf{1 0}(0.12 \mathrm{~g}, 0.32 \mathrm{mmol})$ in methanol $(10 \mathrm{~mL})$ at $0{ }^{\circ} \mathrm{C}$. After 10 min, the suspension was warmed to room temperature and stirred for $1 \mathrm{~h}$. Then the solid was filtered off and washed with $\mathrm{MeOH}$; Py was added until $\mathrm{pH}$ 8. $\mathrm{MeOH}$ was evaporated under reduced pressure and replaced by Py $(3 \mathrm{~mL})$; to the solution was added $\mathrm{Ac}_{2} \mathrm{O}$ at room temperature $(0.04 \mathrm{~mL}, 0.40 \mathrm{mmol})$. After 3 h, solvent removal under reduced pressure and chromatography of the crude residue over silica gel $\left(\mathrm{CH}_{2} \mathrm{Cl}_{2}\right)$ afforded the pure 12, beside to a minor amount of its $\beta$ anomer $(0.12 \mathrm{~g}, 97 \%$ overall yield; 85:15 dr): white solid, mp 70.3-71.8 ${ }^{\circ} \mathrm{C}$ (from $\left.\mathrm{MeOH}\right),[\alpha]_{\mathrm{D}}^{25}-12.0\left(\right.$ c 0.48, $\left.\mathrm{CHCl}_{3}\right) .{ }^{1} \mathrm{H}$ NMR (400 MHz, $\left.\mathrm{CDCl}_{3}\right): \delta 2.10$ (s, 3H), 3.07-3.14 (m, 1H), 3.20-3.30 (m, 2H), 3.32-3.38 (m, 1H), 3.42 (s, 3H), 4.19 (d, $J$ $=9.8 \mathrm{~Hz}, 1 \mathrm{H}), 4.25(\mathrm{dd}, J=11.7, J=4.9 \mathrm{~Hz}, 1 \mathrm{H}), 4.28-4.34(\mathrm{~m}, 1 \mathrm{H}), 4.36(\mathrm{dd}, J=11.7, J=2.0 \mathrm{~Hz}$, 1H), 4.59 (d, $J=11.2 \mathrm{~Hz}, 1 \mathrm{H}), 4.70(\mathrm{~d}, J=11.2 \mathrm{~Hz}, 1 \mathrm{H}), 4.73(\mathrm{~s}, 1 \mathrm{H}), 7.30-7.43(\mathrm{~m}, 5 \mathrm{H}) .{ }^{13} \mathrm{C}$ NMR $(50$ $\left.\mathrm{MHz}, \mathrm{CDCl}_{3}\right): \delta 22.6,29.3,30.0,55.8,63.3,68.0,71.9,74.3,98.1,122.7,126.7,128.0,128.4,130.9$, 137.5, 170.7. Anal. calcd for $\mathrm{C}_{18} \mathrm{H}_{22} \mathrm{O}_{4} \mathrm{~S}_{2}$ : C, 58.99; H, 6.05. Found: C, 59.15; H, 6.07.

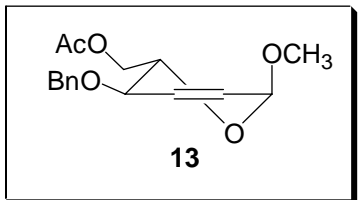

Compound 13. A solution of $12(0.1 \mathrm{~g}, 0.26 \mathrm{mmol})$ in THF ( $3 \mathrm{~mL})$ was added in one portion to a stirred suspension of Raney-Ni (W2) (0.9 g, wet) in the same solvent (3 mL) at $0{ }^{\circ} \mathrm{C}$ and under nitrogen atmosphere. The suspension was stirred for $2 \mathrm{~h}$, then the solid was filtered off and washed with THF. The filtrate was evaporated under reduced pressure to afford a crude residue which chromatography 
over silica gel $\left(\mathrm{CH}_{2} \mathrm{Cl}_{2}\right)$ gave the pure 13 (0.06 g, 75\% yield) beside a small amount of $\mathbf{1 4}(0.01 \mathrm{~g}, 11 \%$ yield). Data for compound 13: oily, $[\alpha]_{\mathrm{D}}^{25}-156.1$ ( $c$ 0.14, MeOH). ${ }^{1} \mathrm{H}$ NMR (400 MHz, $\left.\mathrm{CDCl}_{3}\right): \delta 2.04$ (s, 3H), 3.43 (s, 3H), 4.00 (bs, 2H), 4.30 (bs, 2H), 4.50 (d, $J=11.7 \mathrm{~Hz}, 1 \mathrm{H}), 4.69$ (d, $J=11.7 \mathrm{~Hz}, 1 \mathrm{H}$ ), $4.88(\mathrm{~d}, J=2.4 \mathrm{~Hz}, 1 \mathrm{H}), 5.79(\mathrm{dd}, J=10.7, J=2.4 \mathrm{~Hz}, 1 \mathrm{H}), 6.10(\mathrm{~d}, J=10.2 \mathrm{~Hz}, 1 \mathrm{H}), 7.28-7.40$ (m, 5H). ${ }^{13} \mathrm{C}$ NMR (100 MHz, $\left.\mathrm{CDCl}_{3}\right): \delta 20.7,55.7,63.4,67.6,69.9,70.6,95.5,126.6,127.8,127.9,128.4$, 130.0, 137.5, 170.7. Anal. calcd for $\mathrm{C}_{16} \mathrm{H}_{20} \mathrm{O}_{5}$ : C, 65.74; H, 6.90. Found: C, 65.91; H, 6.88.

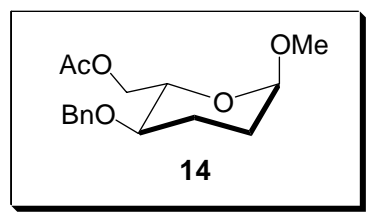

Compound 14. Under similar conditions reported above, treatment of 12 ( $0.1 \mathrm{~g}, 0.26 \mathrm{mmol})$ with an excess of Raney-Ni (W2) (1.8 g, wet) afforded, after common work-up and purification procedures, the pure 14 (0.06 g, 84\% yield): oily, $[\alpha]_{\mathrm{D}}^{25}-36.8$ (c 0.078, MeOH). ${ }^{1} \mathrm{H}$ NMR (400 MHz, $\left.\mathrm{CDCl}_{3}\right): \delta 1.66-$ $1.88(\mathrm{~m}, 4 \mathrm{H}), 2.04$ (s, 3H), 3.27-3.40 (m, 4H), 3.83 (ddd, $J=9.5, J=4.5, J=3.1 \mathrm{~Hz}, 1 \mathrm{H}), 4.29(\mathrm{dd}, J=$ $12.0, J=3.0 \mathrm{~Hz}, 1 \mathrm{H}), 4.31(\mathrm{dd}, J=12.0, J=5.0 \mathrm{~Hz}, 1 \mathrm{H}), 4.43(\mathrm{~d}, J=11.7 \mathrm{~Hz}, 1 \mathrm{H}), 4.63(\mathrm{~d}, J=11.7$ $\mathrm{Hz}, 1 \mathrm{H}), 4.68-4.71(\mathrm{~m}, 1 \mathrm{H}), 7.24-7.40(\mathrm{~m}, 5 \mathrm{H}) .{ }^{13} \mathrm{C} \mathrm{NMR}\left(100 \mathrm{MHz}, \mathrm{CDCl}_{3}\right): \delta 20.7,23.5,28.6,54.2$, 64.0, 69.6, 70.3, 72.4, 97.3, 127.6, 127.7, 127.8, 128.3, 128.4, 138.2, 170.2. Anal. calcd for $\mathrm{C}_{16} \mathrm{H}_{22} \mathrm{O}_{5}$ : C, 65.29; H, 7.53. Found: C, 65.13; H, 7.51.

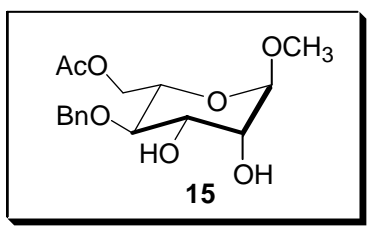

Methyl 6-O-acetyl-4- $\boldsymbol{O}$-benzyl- $\boldsymbol{\alpha}$-L-mannopyranoside (15). To a solution of $\mathbf{1 3}$ (0.06 g, $0.21 \mathrm{mmol})$ in 1:1 (v/v) tert-butyl alcohol/acetone $(2.4 \mathrm{~mL})$, cooled at $0{ }^{\circ} \mathrm{C}$ in ice bath, an excess of a chilled 1:1 (w/w) solution of 4-methylmorpholine- $N$-oxide $(0.06 \mathrm{~g}, 0.42 \mathrm{mmol})$ in water was added in one portion. After 
few minutes, a catalytic amount $(0.04 \mathrm{mmol})$ of $\mathrm{OsO}_{4}$ was added, and the resulting mixture was stirred overnight at room temperature; then the reaction was quenched with saturated aq $\mathrm{Na}_{2} \mathrm{SO}_{3}$ and evaporated under reduced pressure. Chromatography of the crude residue over silica gel (hexane/ acetone, 1:1) afforded the diol 15 (0.06 g, 82\% yield) as a single diastereomer: oily, $[\alpha]_{\mathrm{D}}^{25}-41.0(c 0.29$, $\mathrm{CHCl}_{3}$ ). ${ }^{1} \mathrm{H}$ NMR (400 MHz, $\mathrm{CDCl}_{3}$ ): $\delta 2.08$ (s, 3H), 2.28 (bs, 2H, exchange with $\mathrm{D}_{2} \mathrm{O}$ ), 3.37 (s, 3H), 3.62 (dd, $J=9.8, J=8.8 \mathrm{~Hz}, 1 \mathrm{H}), 3.80$ (ddd, $J=9.8, J=5.1, J=2.2 \mathrm{~Hz}, 1 \mathrm{H}), 3.91-3.97$ (m, $2 \mathrm{H}$ ), 4.30 (dd, $J=11.8, J=5.1 \mathrm{~Hz}, 1 \mathrm{H}), 4.38$ (dd, $J=11.8, J=2.2 \mathrm{~Hz}, 1 \mathrm{H}), 4.69(\mathrm{~d}, J=11.3 \mathrm{~Hz}, 1 \mathrm{H}), 4.73(\mathrm{~d}, J=$ $1.6 \mathrm{~Hz}, 1 \mathrm{H}), 4.76$ (d, $J=11.3 \mathrm{~Hz}, 1 \mathrm{H}), 7.27-7.40$ (m, 5H). ${ }^{13} \mathrm{C} \mathrm{NMR}\left(100 \mathrm{MHz}, \mathrm{CDCl}_{3}\right): \delta$ 20.9, 55.0, 63.4, 69.0, 71.0, 71.8, 74.8, 75.7, 100.5, 124.5, 128.1, 128.7, 137.9, 170.8. Anal. calcd for $\mathrm{C}_{16} \mathrm{H}_{22} \mathrm{O}_{7}$ : C, 58.89; H, 6.79. Found: C, 59.05; H, 6.77.

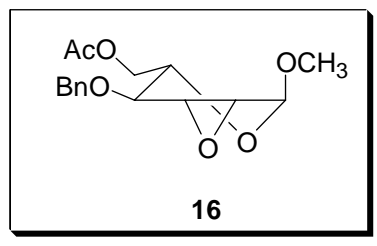

Compound 16. $\mathrm{Na}_{2}$ EDTA $\left(4.0 \times 10^{-4} \mathrm{M}, 0.60 \mathrm{~mL}\right)$ and $\mathrm{CF}_{3} \mathrm{COCH}_{3}(0.11 \mathrm{~mL})$ were added to a solution of $13(0.03 \mathrm{~g}, 0.12 \mathrm{mmol})$ in $\mathrm{CH}_{3} \mathrm{CN}(1.25 \mathrm{~mL})$ at $0{ }^{\circ} \mathrm{C}$. After a few minutes a mixture of $\mathrm{NaHCO}_{3}(0.08$ g) and Oxone ( $0.37 \mathrm{~g})$ was added over $1 \mathrm{~h}$ and the whole resulting mixture was stirred for $30 \mathrm{~min}$ at the same temperature. Then the reaction was diluted with $\mathrm{H}_{2} \mathrm{O}$ and extracted with $\mathrm{CH}_{2} \mathrm{Cl}_{2}$. The extracts were washed with brine, dried $\left(\mathrm{Na}_{2} \mathrm{SO}_{4}\right)$, and evaporated under reduced pressure. Chromatography of the crude residue over silica gel (hexane/acetone $=8: 2)$ afforded the pure $\mathbf{1 6}(0.03 \mathrm{~g}, 92 \%$ yield $)$ as a single diastereoisomer: oily, $[\alpha]_{\mathrm{D}}^{25}-107.3\left(c\right.$ 0.33, $\left.\mathrm{CHCl}_{3}\right) .{ }^{1} \mathrm{H} \mathrm{NMR}\left(500 \mathrm{MHz}, \mathrm{CDCl}_{3}\right): \delta 2.00$ (s, 3H), 3.10 (d, $J=3.9 \mathrm{~Hz}, 1 \mathrm{H}), 3.37$ (d, $J=3.9 \mathrm{~Hz}, 1 \mathrm{H}), 3.45$ (s, 3H), 3.54 (d, $J=9.8 \mathrm{~Hz}, 1 \mathrm{H}), 3.76$ (ddd, $J=$ 9.8, $J=5.4, J=2.5 \mathrm{~Hz}, 1 \mathrm{H}), 4.14$ (dd, $J=11.7, J=5.4 \mathrm{~Hz}, 1 \mathrm{H}), 4.19$ (dd, $J=11.7, J=2.5 \mathrm{~Hz}, 1 \mathrm{H}$ ), 4.55 (d, $J=11.7 \mathrm{~Hz}, 1 \mathrm{H}), 4.78$ (d, $J=11.7 \mathrm{~Hz}, 1 \mathrm{H}), 4.89$ (s, 1H), 7.27-7.40 (m, 5H). ${ }^{13} \mathrm{C}$ NMR (75 MHz, 
$\left.\mathrm{CDCl}_{3}\right): \delta 20.7,49.5,53.0,55.4,63.2,65.5,68.3,71.7,96.0,128.1,128.5,128.6,136.8,171.0$. Anal. calcd for $\mathrm{C}_{16} \mathrm{H}_{20} \mathrm{O}_{6}$ : C, 62.33; H, 6.54. Found: C, 62.12; H, 6.56.

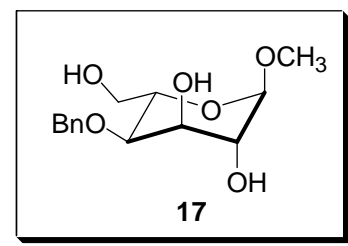

Methyl-4-O-benzyl- $\boldsymbol{\alpha}$-L-altropyranoside (17). Method A: the epoxide 16 (0.03 g, $0.09 \mathrm{mmol})$ was refluxed for 5 days in a $0.1 \mathrm{M}$ aq solution of $\mathrm{KOH}(1 \mathrm{ml})$. After solvent removal under reduced pressure, chromatography of the crude residue over silica gel $\left(\mathrm{CHCl}_{3} / \mathrm{MeOH}=95 / 5\right)$ afforded the pure 17 (0.02 g, 90 \% yield). Method B: a solution of the epoxide 16 (0.03 g, $0.09 \mathrm{mmol})$ in an aq solution of $6 \% \mathrm{HClO}_{4}(0.6 \mathrm{~mL})$ was stirred for $16 \mathrm{hr}$ at room temperature. Solid $\mathrm{Na}_{2} \mathrm{CO}_{3}$ was added until $\mathrm{pH}$ 8, then solvent removal under reduced pressure afforded 17 (0.02 g, $95 \%$ yield): oily, $[\alpha]_{\mathrm{D}}^{25}-21.08$ (c 2.0, $\mathrm{CHCl}_{3}$ ). ${ }^{1} \mathrm{H}$ NMR (500 MHz, $\mathrm{CDCl}_{3}$ ): $\delta 1.90$ (bs, 3H, $\mathrm{D}_{2} \mathrm{O}$ exchange), 3.42 (s, 3H), 3.78-3.84 (m, 2H), 3.87 (dd, $J=12.2, J=3.2 \mathrm{~Hz}, 1 \mathrm{H}$ ), 3.92-3.99 (m, 2H), 4.12 (bs, 1H), 4.56 (d, $J=11.2 \mathrm{~Hz}, 1 \mathrm{H}), 4.68$ (s, 1H), 4.71 (d, $J=11.7 \mathrm{~Hz}, 1 \mathrm{H}), 7.28-7.40(\mathrm{~m}, 5 \mathrm{H}) .{ }^{13} \mathrm{C}$ NMR $\left(125 \mathrm{MHz}, \mathrm{CDCl}_{3}\right): \delta$ 55.9, 62.7, 67.3, 68.4, 70.0, 71.3, 71.6, 101.7, 128.3, 128.8, 137.8. Anal. calcd for $\mathrm{C}_{14} \mathrm{H}_{20} \mathrm{O}_{6}$ : C, 59.14; $\mathrm{H}, 7.09$. Found: C, 59.01; H, 7.07. 


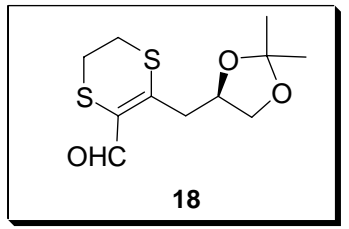

Compound 18. As result of a purification procedure of compound 8 (0.11 g, $0.29 \mathrm{mmol})$, chromatography over silica gel $\left(1.5 \mathrm{~g}, \mathrm{CH}_{2} \mathrm{Cl}_{2}\right)$ afforded the pure $\mathbf{1 8}\left(0.08 \mathrm{~g}, 98 \%\right.$ yield): oily, $[\alpha]_{\mathrm{D}}^{25}$ +30.5 (c 1.0, $\left.\mathrm{CHCl}_{3}\right) .{ }^{1} \mathrm{H}$ NMR (400 MHz, $\left.\mathrm{CDCl}_{3}\right): \delta 1.35$ (s, 3H), 1.42 (s, 3H), 2.72 (dd, $J=14.8, J=$ $4.7 \mathrm{~Hz}, 1 \mathrm{H}$ ), 3.08-3.16 (m, 2H), 3.21 (dd, $J=14.9, J=7.3 \mathrm{~Hz}, 1 \mathrm{H}), 3.25-3.38$ (m, 2H), 3.76 (dd, $J=8.3$, $J=6.5 \mathrm{~Hz}, 1 \mathrm{H}), 4.13(\mathrm{dd}, J=8.3, J=5.8 \mathrm{~Hz}, 1 \mathrm{H}), 4.28-4.38(\mathrm{~m}, 1 \mathrm{H}), 9.90$ (s, $1 \mathrm{H}) .{ }^{13} \mathrm{C}$ NMR $(50 \mathrm{MHz}$, $\left.\mathrm{CDCl}_{3}\right): \delta 25.2,26.5,29.1,29.5,38.7,68.4,78.9,109.7,131.9,159.0,183.4$. Anal. calcd for $\mathrm{C}_{11} \mathrm{H}_{16} \mathrm{O}_{3} \mathrm{~S}_{2}$ : C, 50.74; H, 6.19. Found: C, 50.88; H, 6.21.

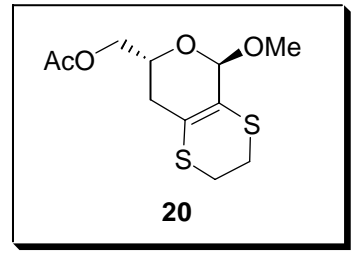

Compound 20. Amberlyst 15 (1.0 g, previously washed with anhydrous $\mathrm{MeOH}$ ), was added in one portion to a stirred solution of aldehyde $18(0.10 \mathrm{~g}, 0.37 \mathrm{mmol})$ in methanol $(9.0 \mathrm{~mL})$ at $0{ }^{\circ} \mathrm{C}$. After 10 min, the suspension was warmed to room temperature and stirred for $1 \mathrm{~h}$. Then the solid was filtered off and washed with $\mathrm{MeOH}$; Py was added until $\mathrm{pH}$ 8. $\mathrm{MeOH}$ was evaporated under reduced pressure and replaced by Py $(3 \mathrm{~mL})$; to the solution was then added $\mathrm{Ac}_{2} \mathrm{O}(0.04 \mathrm{~mL}, 0.45 \mathrm{mmol})$. After $3 \mathrm{~h}$, solvent removal under reduced pressure and chromatography of the crude residue over silica gel $\left(\mathrm{CH}_{2} \mathrm{Cl}_{2}\right)$ afforded the pure 20, beside to a minor amount of its $\beta$-anomer ( $0.10 \mathrm{~g}, 95 \%$ overall yield; 85:15 dr): oily, $[\alpha]_{\mathrm{D}}^{25}+20.6\left(c \mathrm{1} .0, \mathrm{CHCl}_{3}\right) .{ }^{1} \mathrm{H} \mathrm{NMR}\left(500 \mathrm{MHz}, \mathrm{CDCl}_{3}\right): \delta 1.95$ (dd, $\left.J=16.1, J=3.4 \mathrm{~Hz}, 1 \mathrm{H}\right), 2.10$ (s, 3H), 2.34 (dd, $J=16.1, J=11.2 \mathrm{~Hz}, 1 \mathrm{H}), 3.13-3.20$ (m, 2H), 3.21-3.30 (m, 2H), 3.43 (s, 3H), 4.14 
(dd, $J=11.7, J=3.9 \mathrm{~Hz}, 1 \mathrm{H}), 4.18$ (dd, $J=11.7, J=5.9 \mathrm{~Hz}, 1 \mathrm{H}), 4.29-4.35$ (m, $1 \mathrm{H}), 4.74(\mathrm{~m}, 1 \mathrm{H}) .{ }^{13} \mathrm{C}$ NMR (50 MHz, $\left.\mathrm{CDCl}_{3}\right): \delta$ 20.7, 27.3, 28.5, 32.8, 55.2, 66.3, 71.6, 98.2, 127.3, 128.1, 170.7. Anal. calcd for $\mathrm{C}_{11} \mathrm{H}_{16} \mathrm{O}_{4} \mathrm{~S}_{2}$ : C, 47.80; H, 5.84. Found: C, 47.95; H, 5.82.

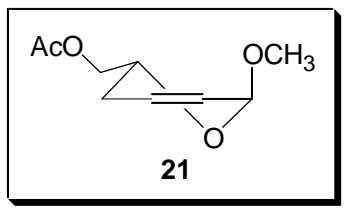

Compound 21. A solution of $20(0.08 \mathrm{~g}, 0.29 \mathrm{mmol})$ in THF $(2.5 \mathrm{~mL})$ was added in one portion to a stirred suspension of Raney-Ni (W2) (0.7 g, wet) in the same solvent $(2.5 \mathrm{~mL})$ at $0{ }^{\circ} \mathrm{C}$ and under nitrogen atmosphere. The suspension was stirred for 1h, then the solid was filtered off and washed with THF. The filtrate was evaporated under reduced pressure to afford a crude residue which chromatography over silica gel $\left(\mathrm{CH}_{2} \mathrm{Cl}_{2}\right)$ gave the pure 21 (0.04 g, 76\% yield): oily. ${ }^{1} \mathrm{H}$ NMR (300 MHz, $\left.\mathrm{CDCl}_{3}\right): \delta$ 1.92-195 (m, 1H), 2.08-2.15 (m, 4H), 3.43 (s, 3H), 4.25-4.32 (m, 3H), 4.88 (d, $J=2.5$ $\mathrm{Hz}, 1 \mathrm{H}), 5.80$ (dd, $J=2.5 \mathrm{~Hz}, J=10.3 \mathrm{~Hz}, 1 \mathrm{H}), 6.10$ (d, $J=10.3 \mathrm{~Hz}, 1 \mathrm{H}) .{ }^{13} \mathrm{C}$ NMR $(125 \mathrm{MHz}$, $\left.\mathrm{CDCl}_{3}\right): \delta 20.7,27.1,55.2,65.3,66.8,95.6,126.8,128.3,170.7$. Anal. calcd for $\mathrm{C}_{9} \mathrm{H}_{14} \mathrm{O}_{4}$ : C, 58.05; $\mathrm{H}$, 7.58. Found: C, 58.30; H, 7.55.

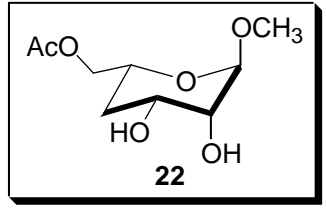

Methyl 6-O-acetyl-4-deoxy- $\alpha$-L-lyxo-hexopyranoside (22). To a solution of 21 (0.04 g, $0.22 \mathrm{mmol}$ ) in 1:1 (v/v) tert-butyl alcohol/acetone $(2.5 \mathrm{~mL})$, cooled at $0{ }^{\circ} \mathrm{C}$ in ice bath, an excess of a chilled 1:1 (w/w) solution of 4-methylmorpholine- $N$-oxide $(0.06 \mathrm{~g}, 0.44 \mathrm{mmol})$ in water was added in one portion. After few minutes, a catalytic amount $(0.04 \mathrm{mmol})$ of $\mathrm{OsO}_{4}$ was added, and the resulting mixture was stirred 
overnight at room temperature; then the reaction was quenched with saturated aq $\mathrm{Na}_{2} \mathrm{SO}_{3}$ and evaporated under reduced pressure. Chromatography of the crude residue over silica gel (hexane/ acetone, 6:4) afforded the diol 22 (0.04 g, 86\% yield) as a single diastereomer: oily. ${ }^{1} \mathrm{H}$ NMR (400 MHz, $\left.\mathrm{CDCl}_{3}\right): \delta$ 1.58-1.68 (m, 1H), 1.73-1.78 (m, 1H), 2.11 (s, 3H), 2.40 (bs, 2H, exchange with $\mathrm{D}_{2} \mathrm{O}$ ), 3.37 (s, 3H), 3.72-3.79 (m, 1H), 3.93-4.01 (m, 2H), 4.26 (dd, $J=4.4 \mathrm{~Hz}, J=10.2 \mathrm{~Hz}, 1 \mathrm{H}), 4.29$ (dd, $J=$ $5.4 \mathrm{~Hz}, J=10.2 \mathrm{~Hz}, 1 \mathrm{H}), 4.78$ (s, 1H). ${ }^{13} \mathrm{C}$ NMR (125 MHz, $\left.\mathrm{CDCl}_{3}\right): \delta$ 20.7, 30.7, 55.1, 63.6, 68.1, 69.8, 101.3, 170.7. Anal. calcd for $\mathrm{C}_{9} \mathrm{H}_{16} \mathrm{O}_{6}$ : C, 49.09; H, 7.32. Found: C, 49.31; H, 7.30. 
$500 \mathrm{MHz}, \mathrm{CDCl}_{3}$
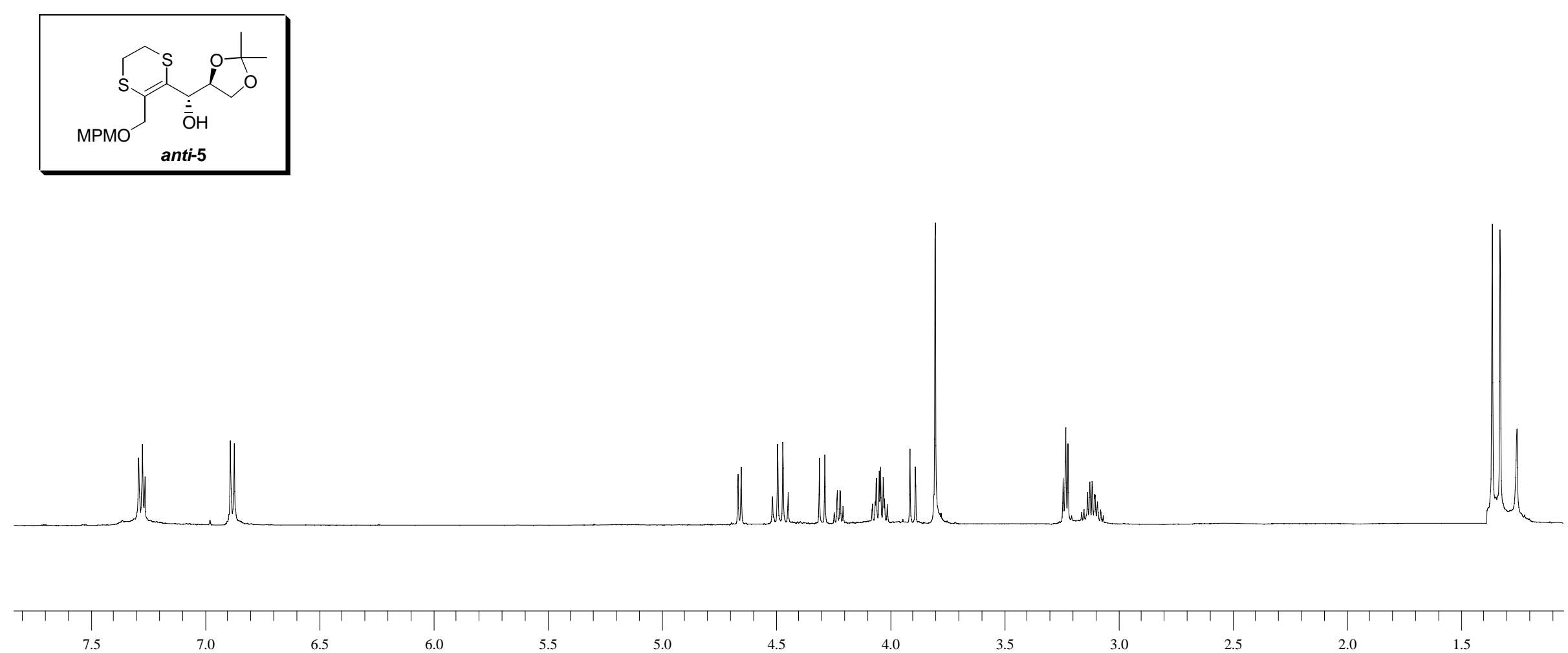
$500 \mathrm{MHz}, \mathrm{CDCl}_{3}$
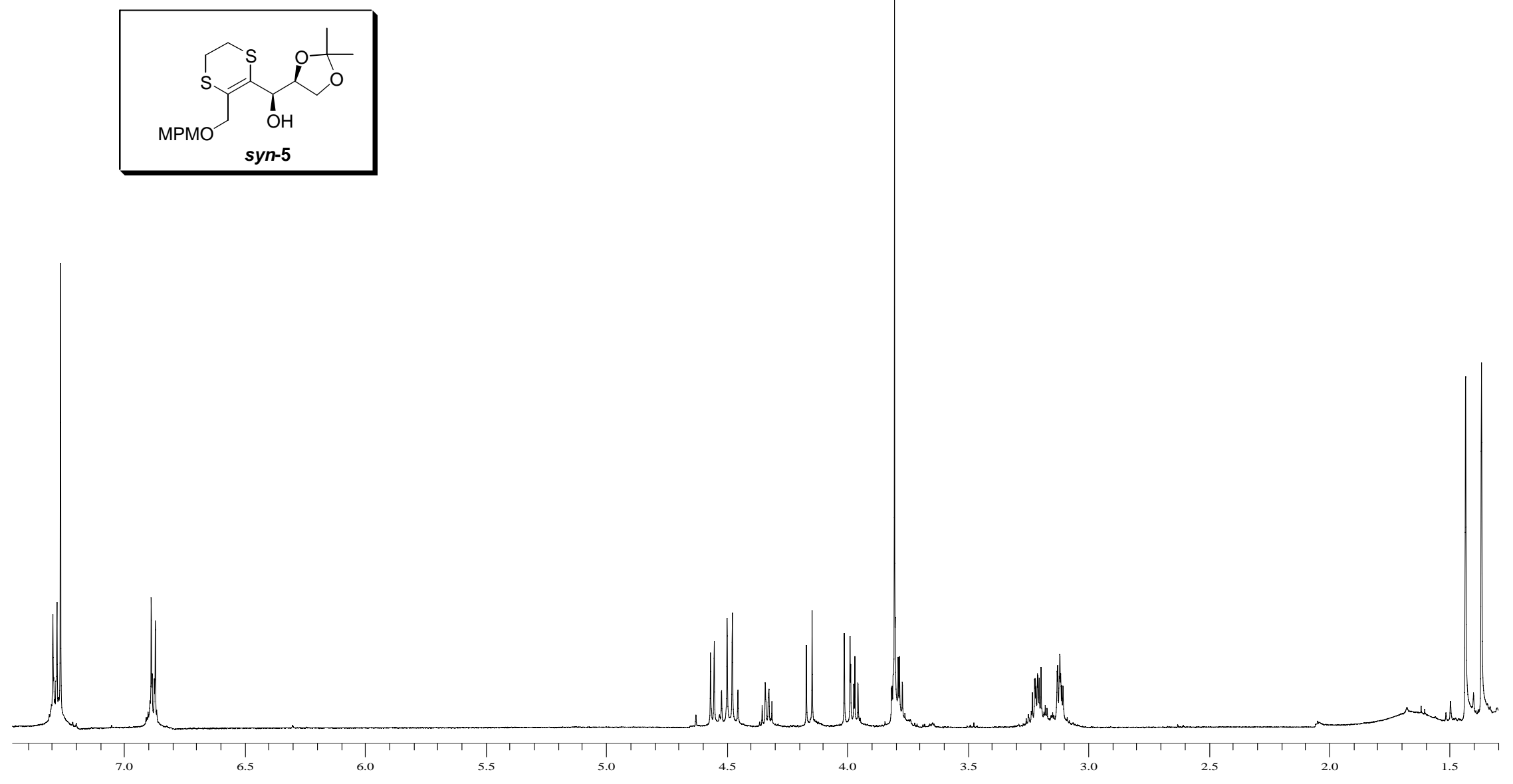


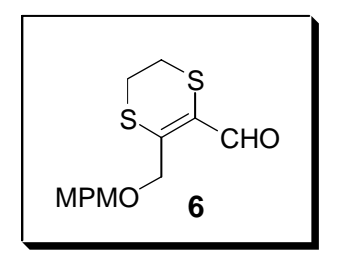

$500 \mathrm{MHz}, \mathrm{CDCl}_{3}$

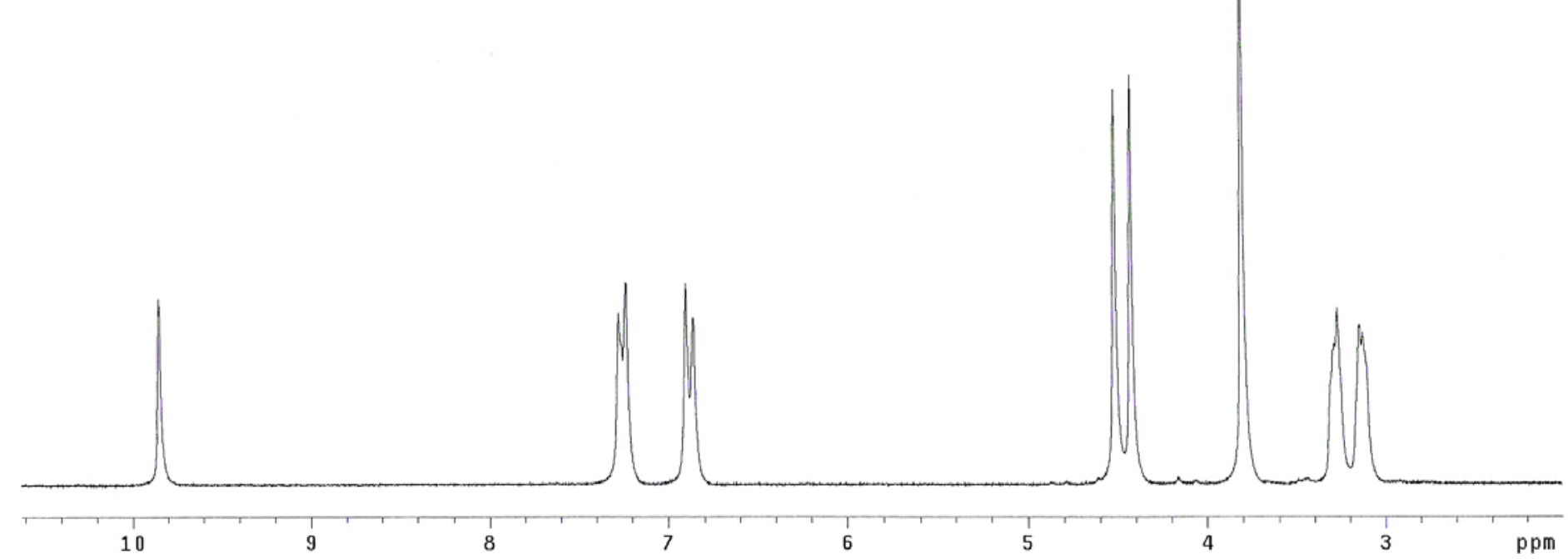


$500 \mathrm{MHz}, \mathrm{CDCl}_{3}$

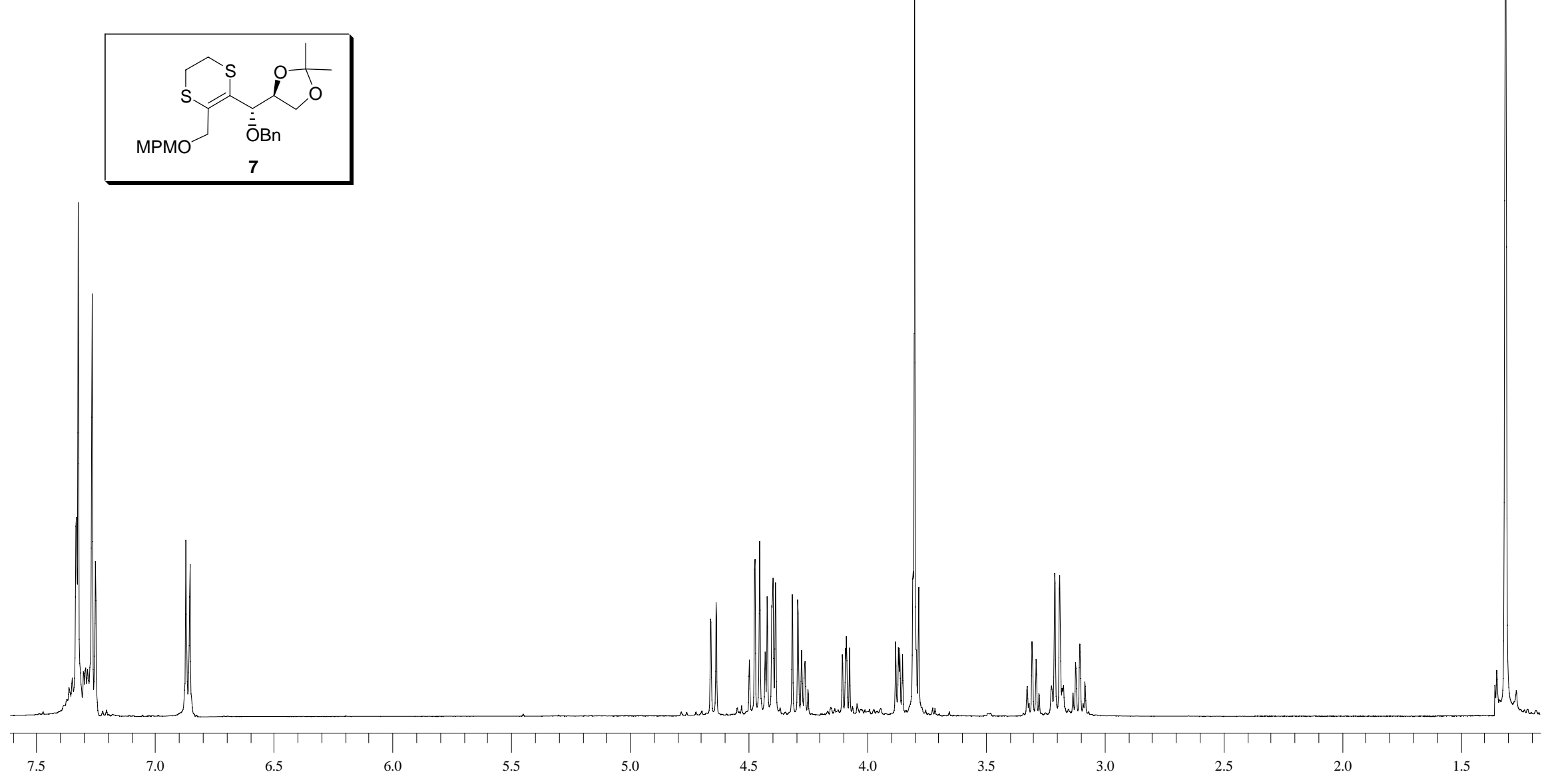




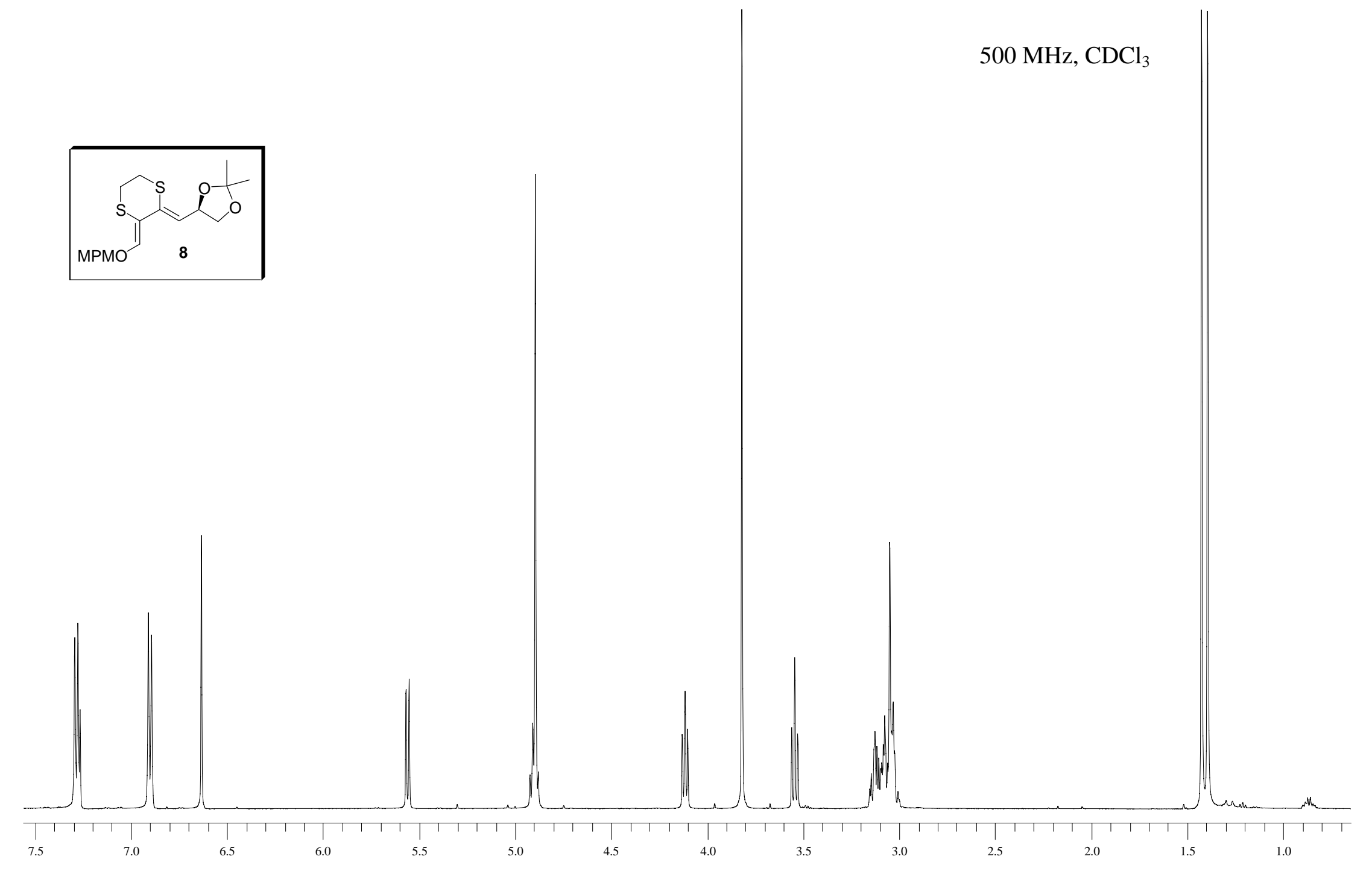


$500 \mathrm{MHz}, \mathrm{CDCl}_{3}$
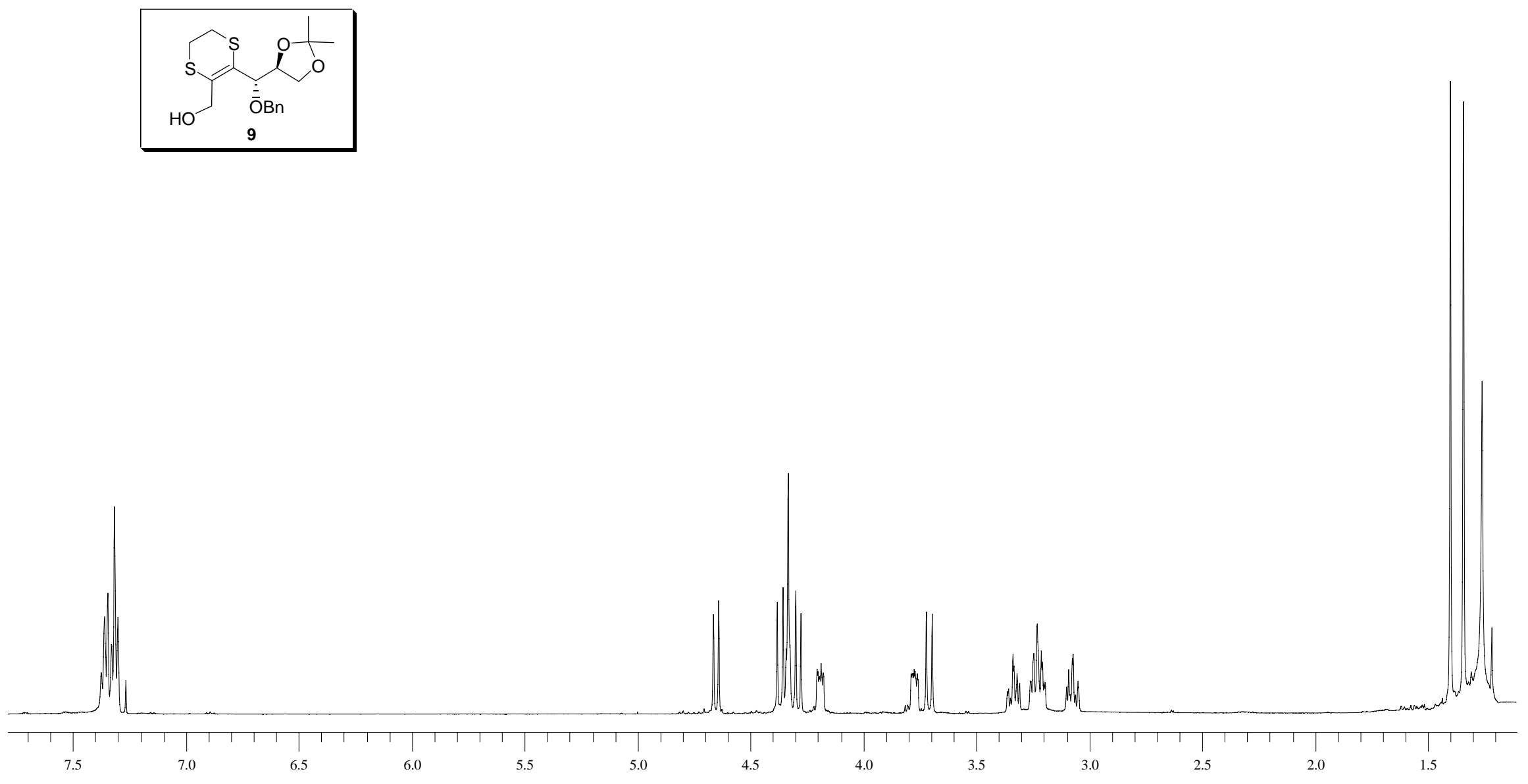


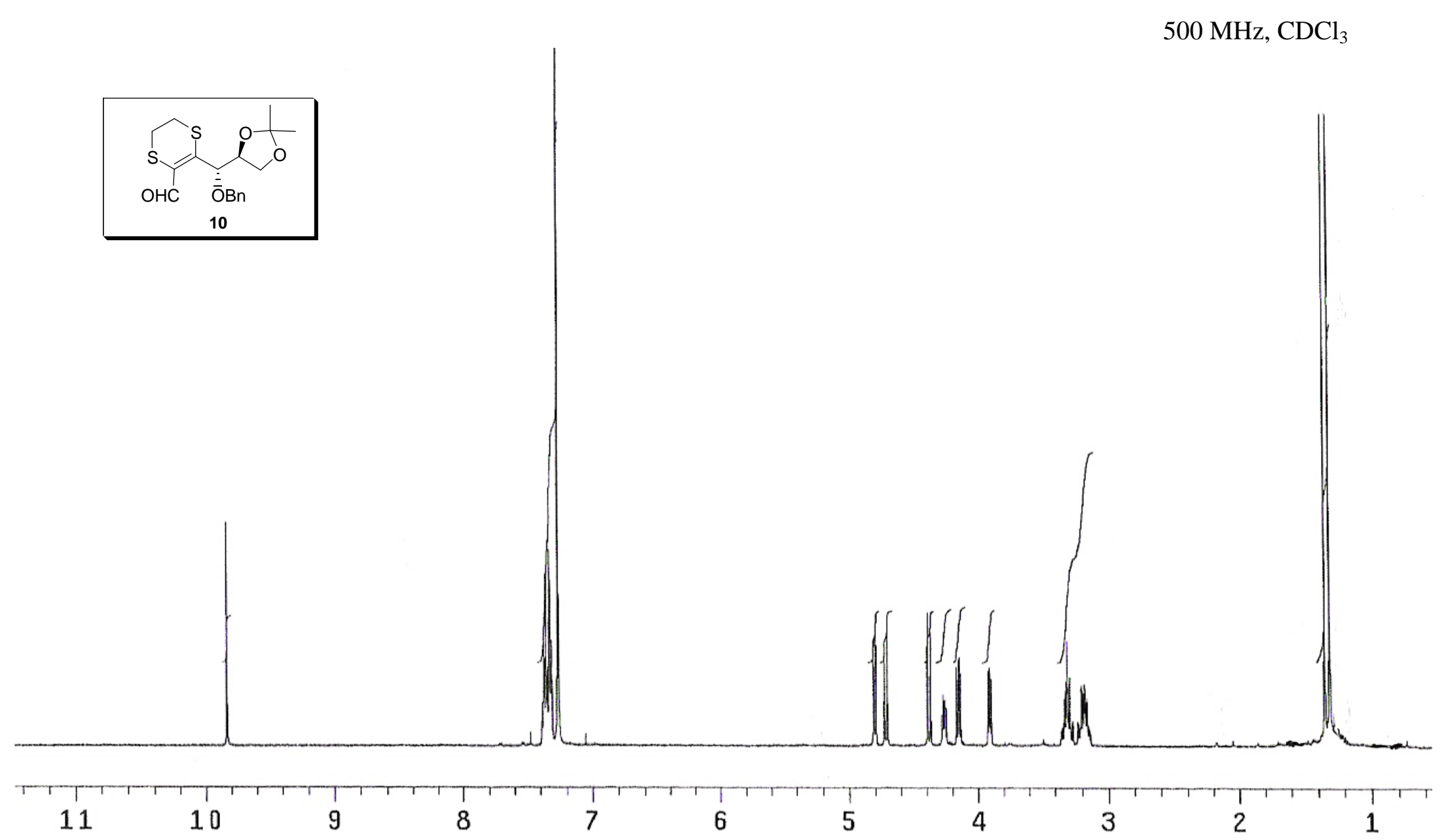




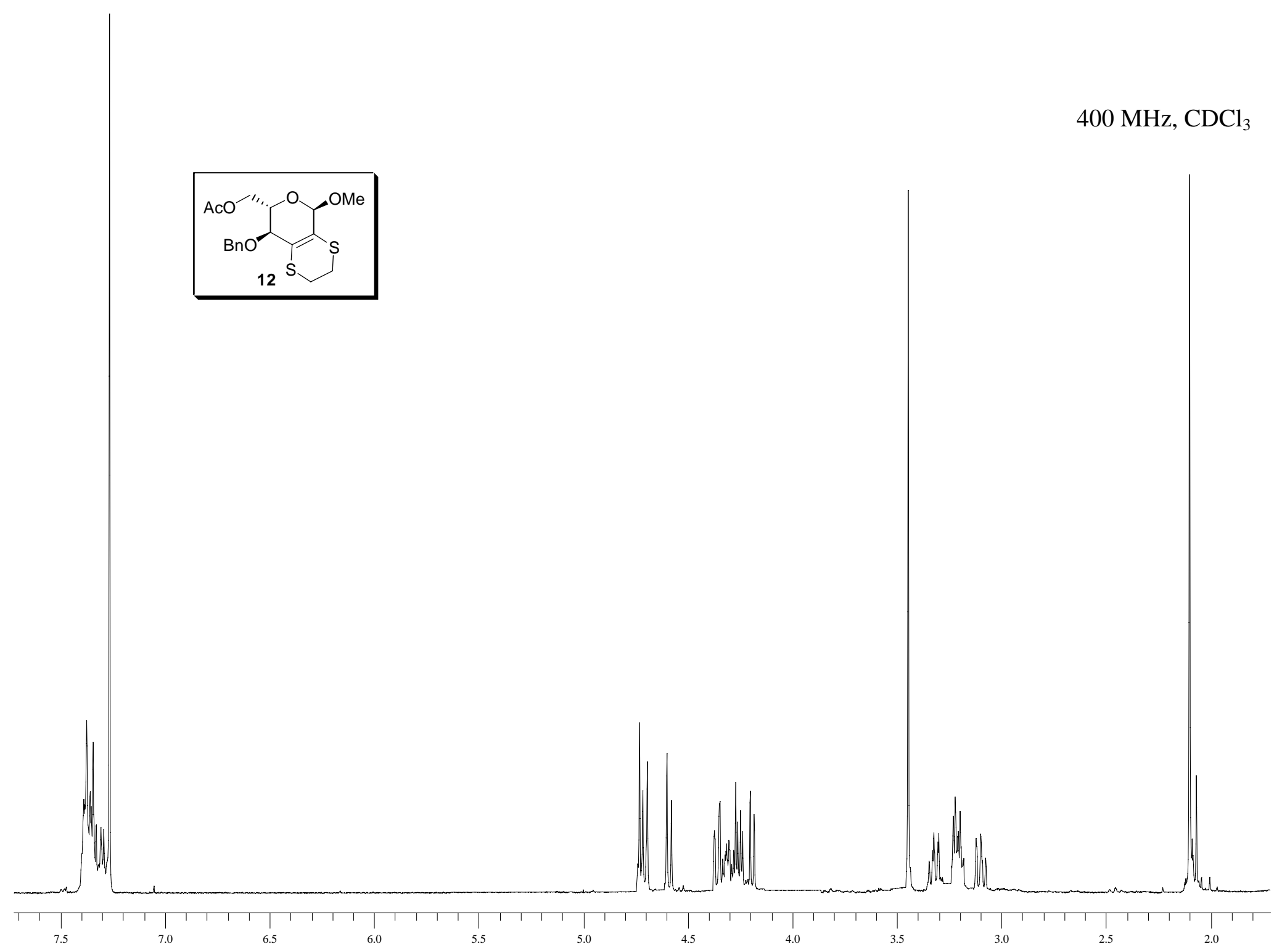




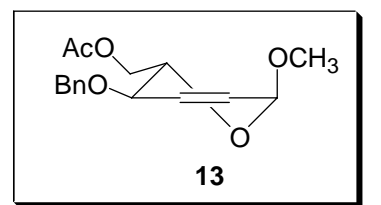

$400 \mathrm{MHz}, \mathrm{CDCl}_{3}$

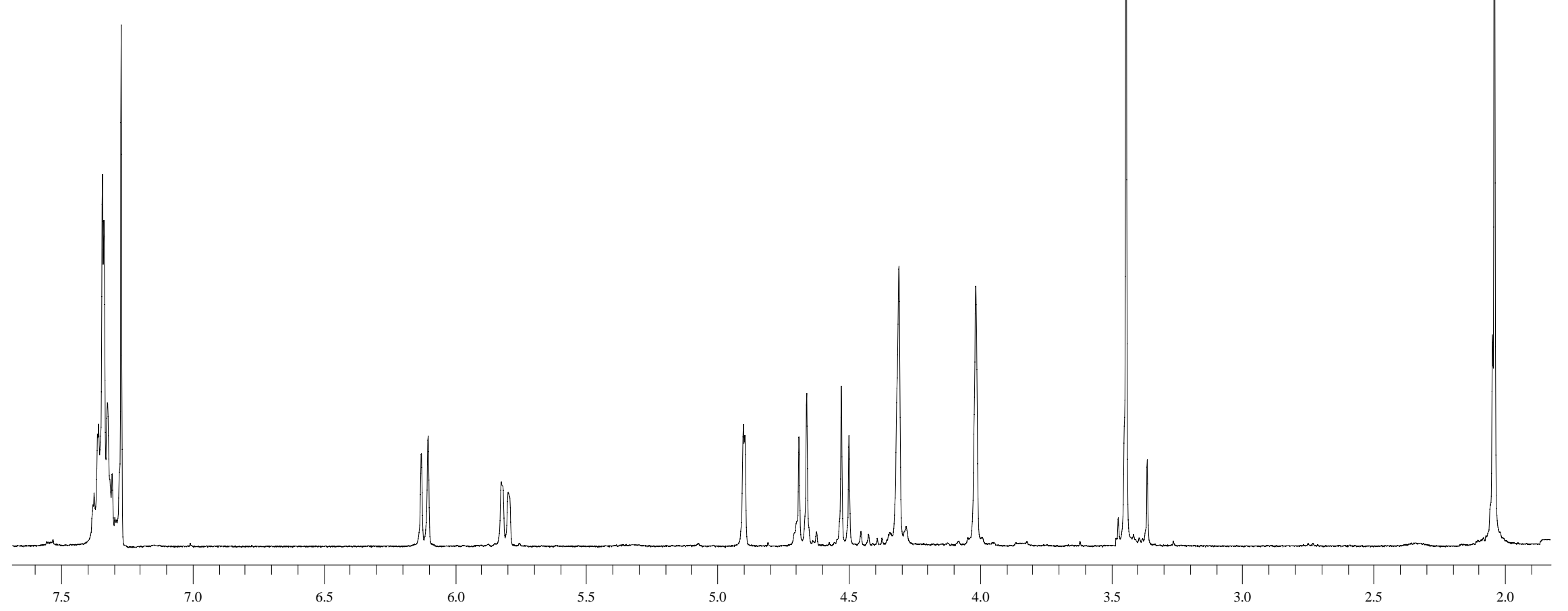




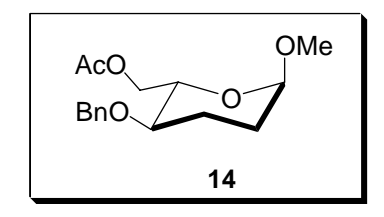

$400 \mathrm{MHz}, \mathrm{CDCl}_{3}$

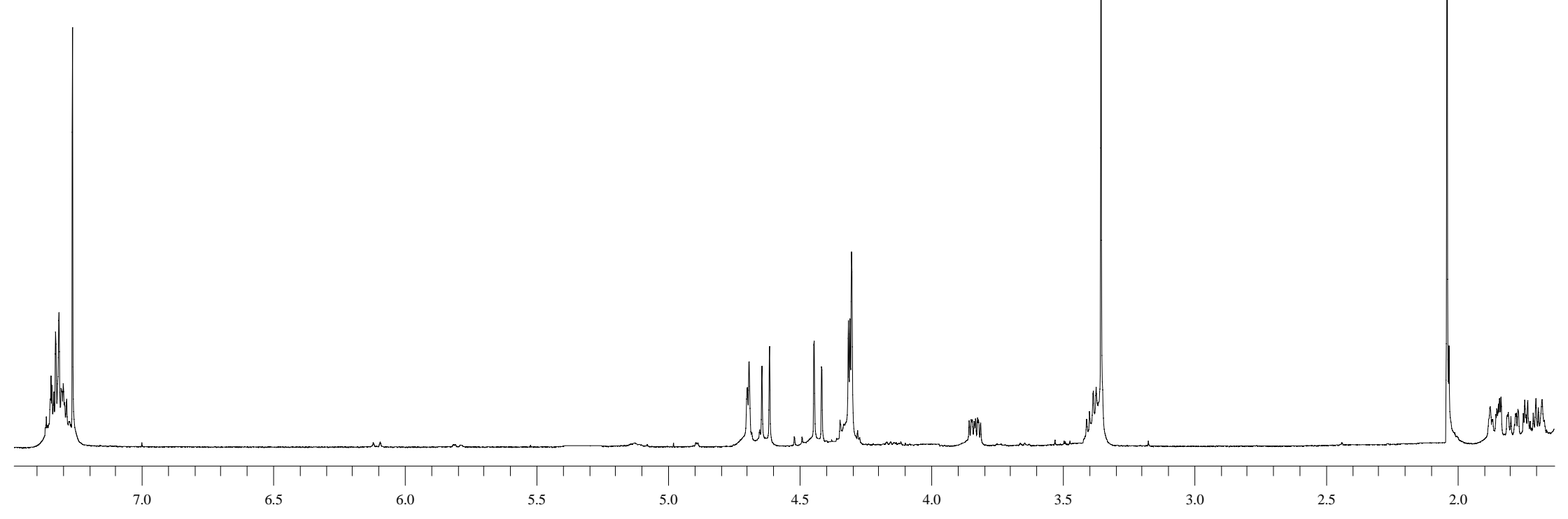




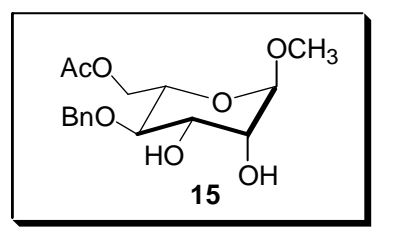

$400 \mathrm{MHz} \mathrm{CDCl}_{3}$

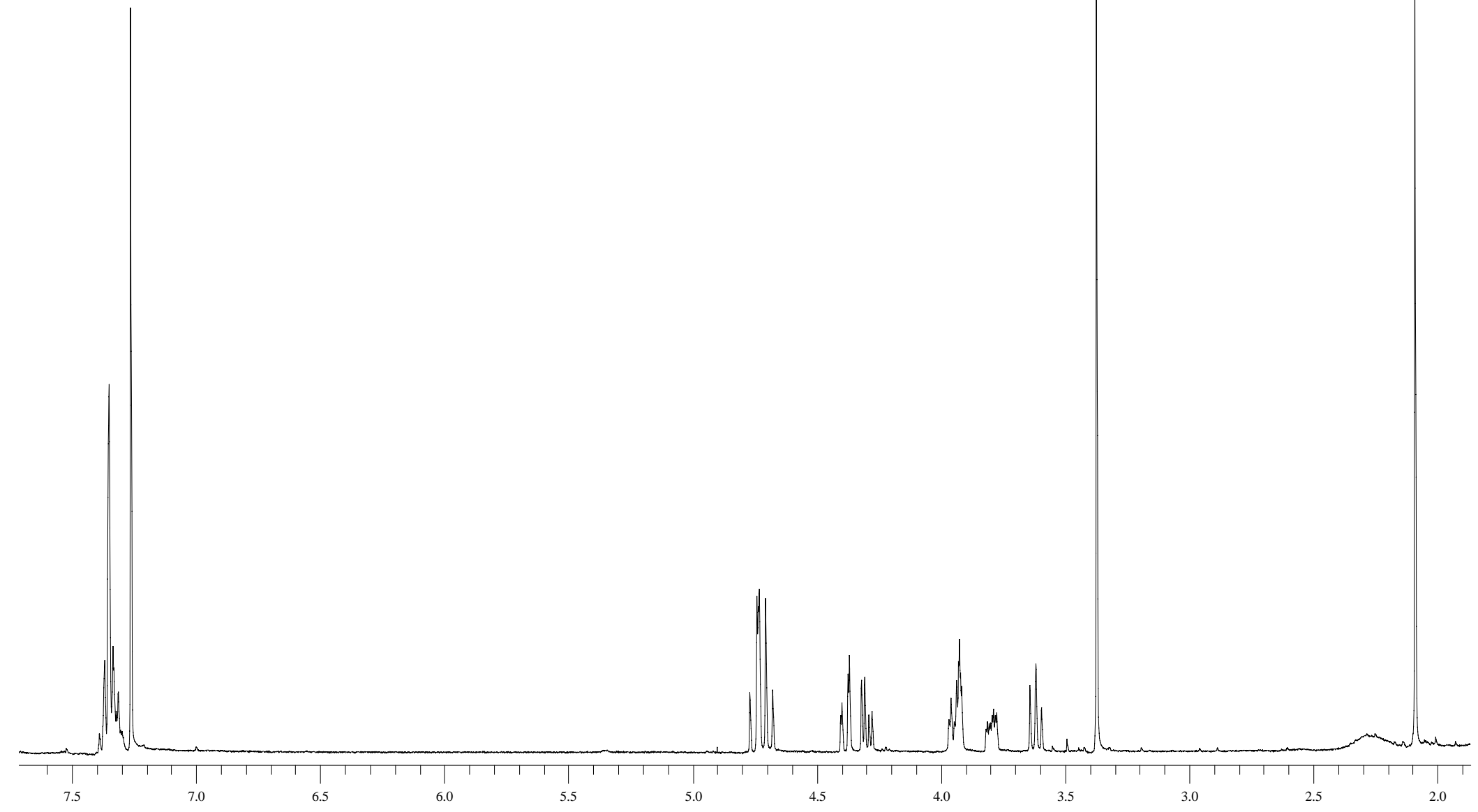




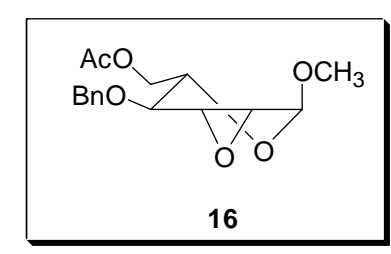

$500 \mathrm{MHz}, \mathrm{CDCl}_{3}$

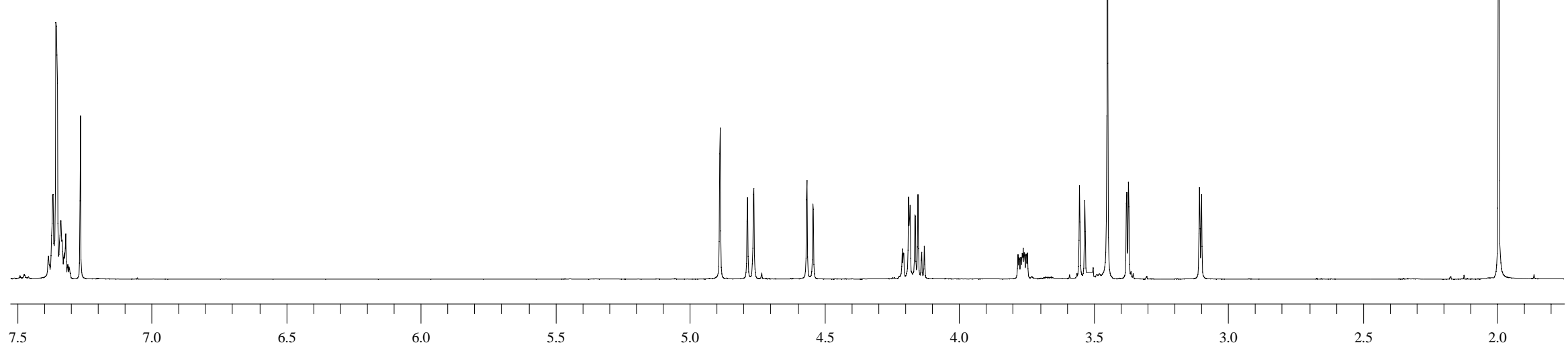




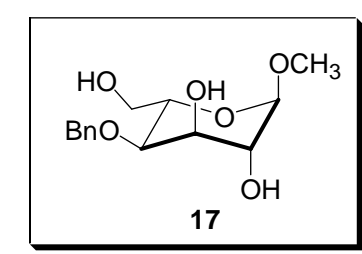

$500 \mathrm{MHz}, \mathrm{CDCl}_{3}$

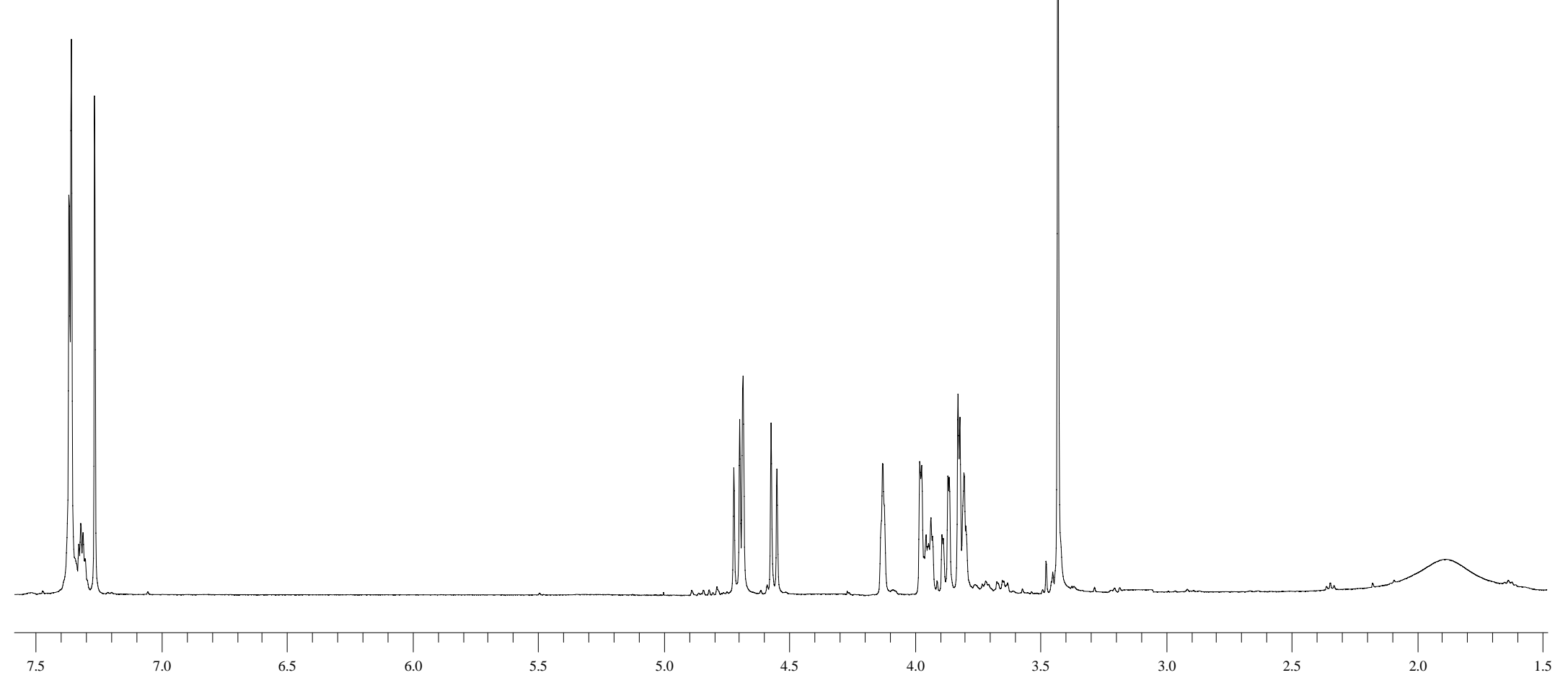


$400 \mathrm{MHz}, \mathrm{CDCl}_{3}$

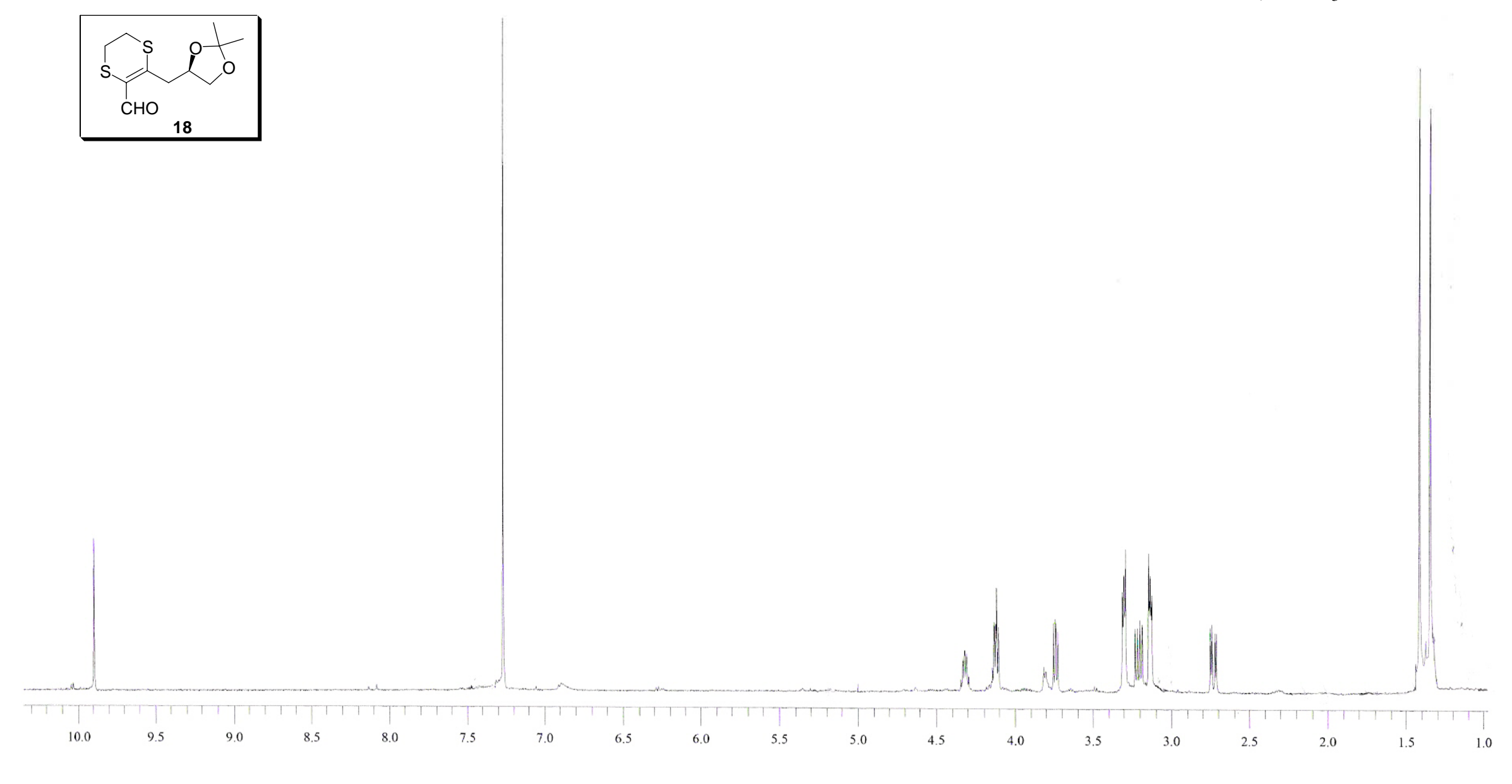




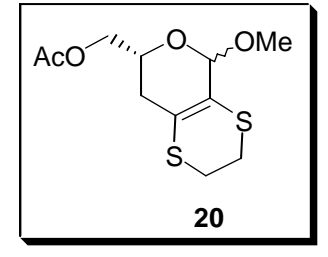

$500 \mathrm{MHz}, \mathrm{CDCl}_{3}$

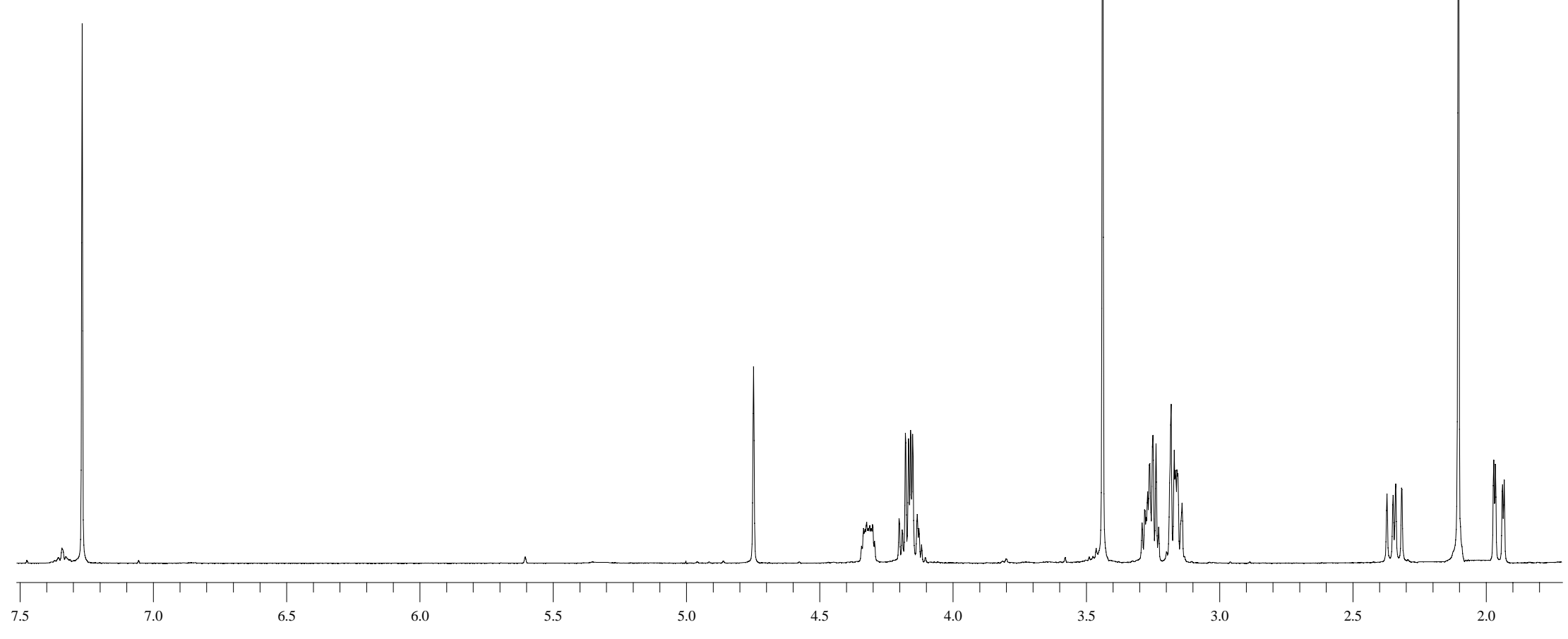


$300 \mathrm{MHz}, \mathrm{CDCl}_{3}$
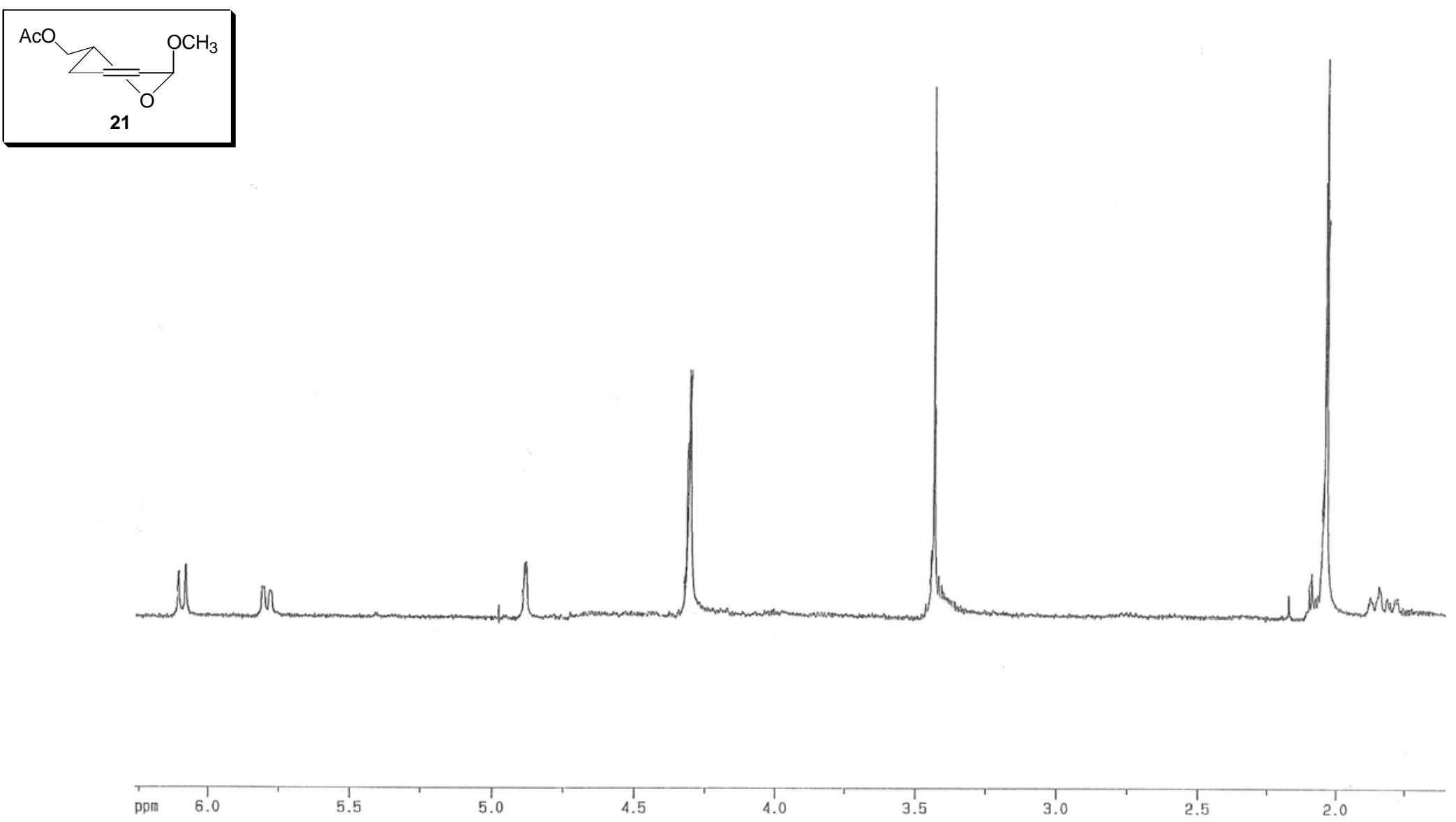


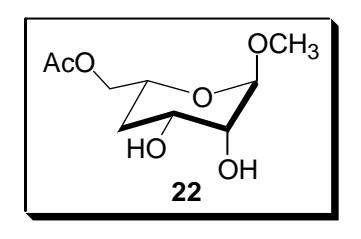

$400 \mathrm{MHz}, \mathrm{CDCl}_{3}$

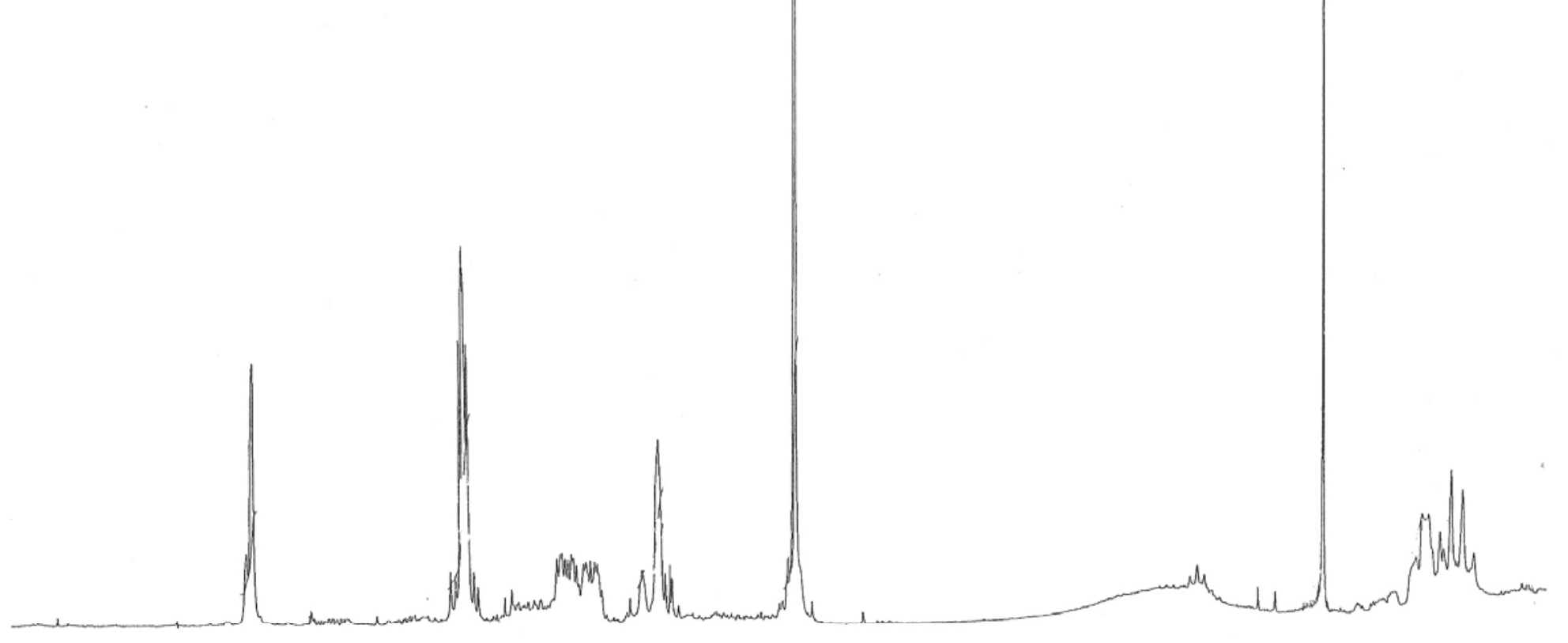

ppm

5.0

4.5

${ }_{3}^{1} \cdot 5$

3.0

2.5

2.0 


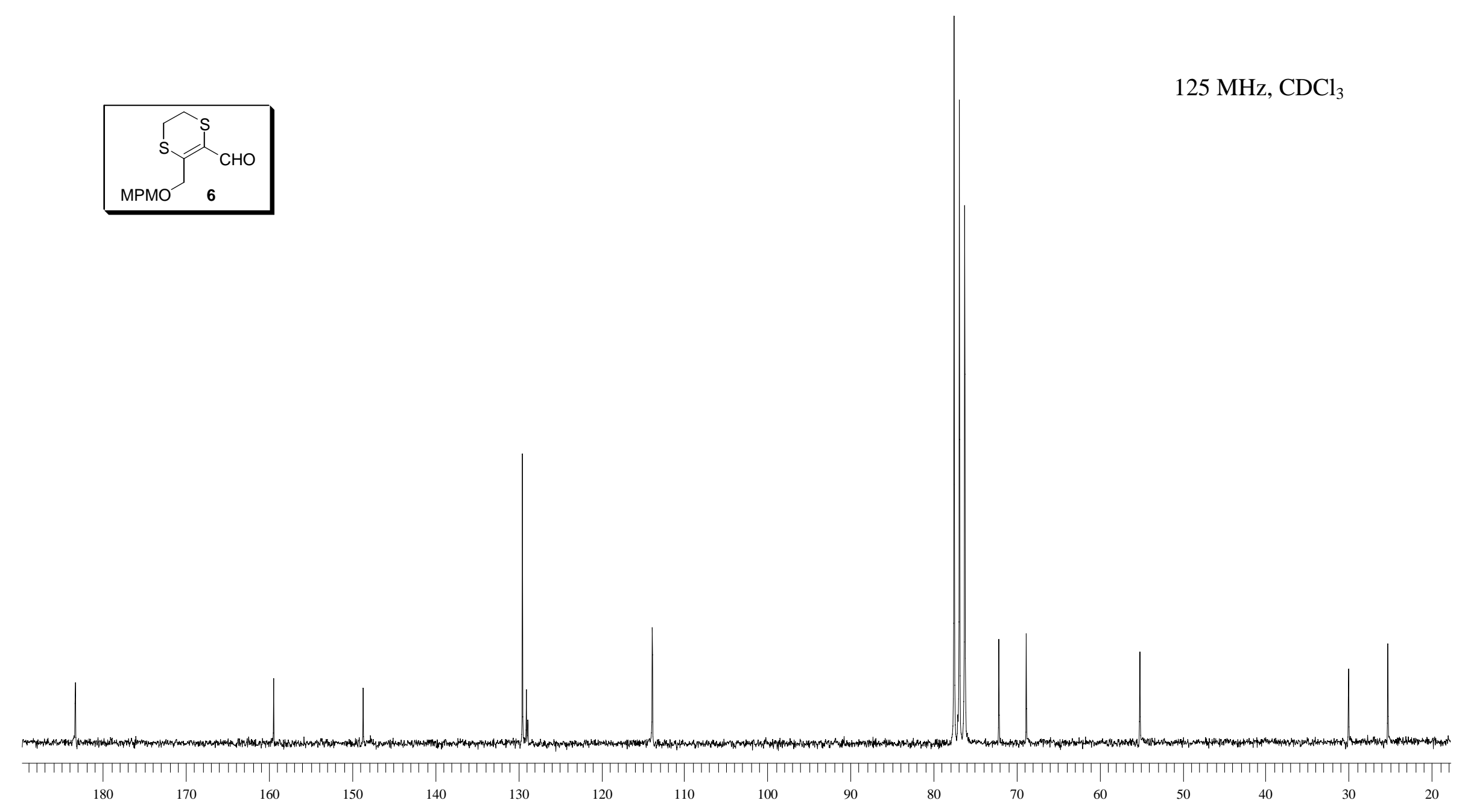




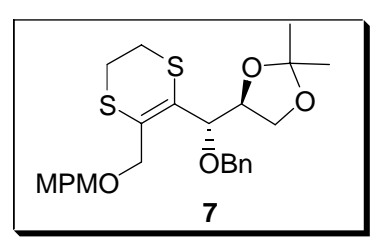

$125 \mathrm{MHz}, \mathrm{CDCl}_{3}$
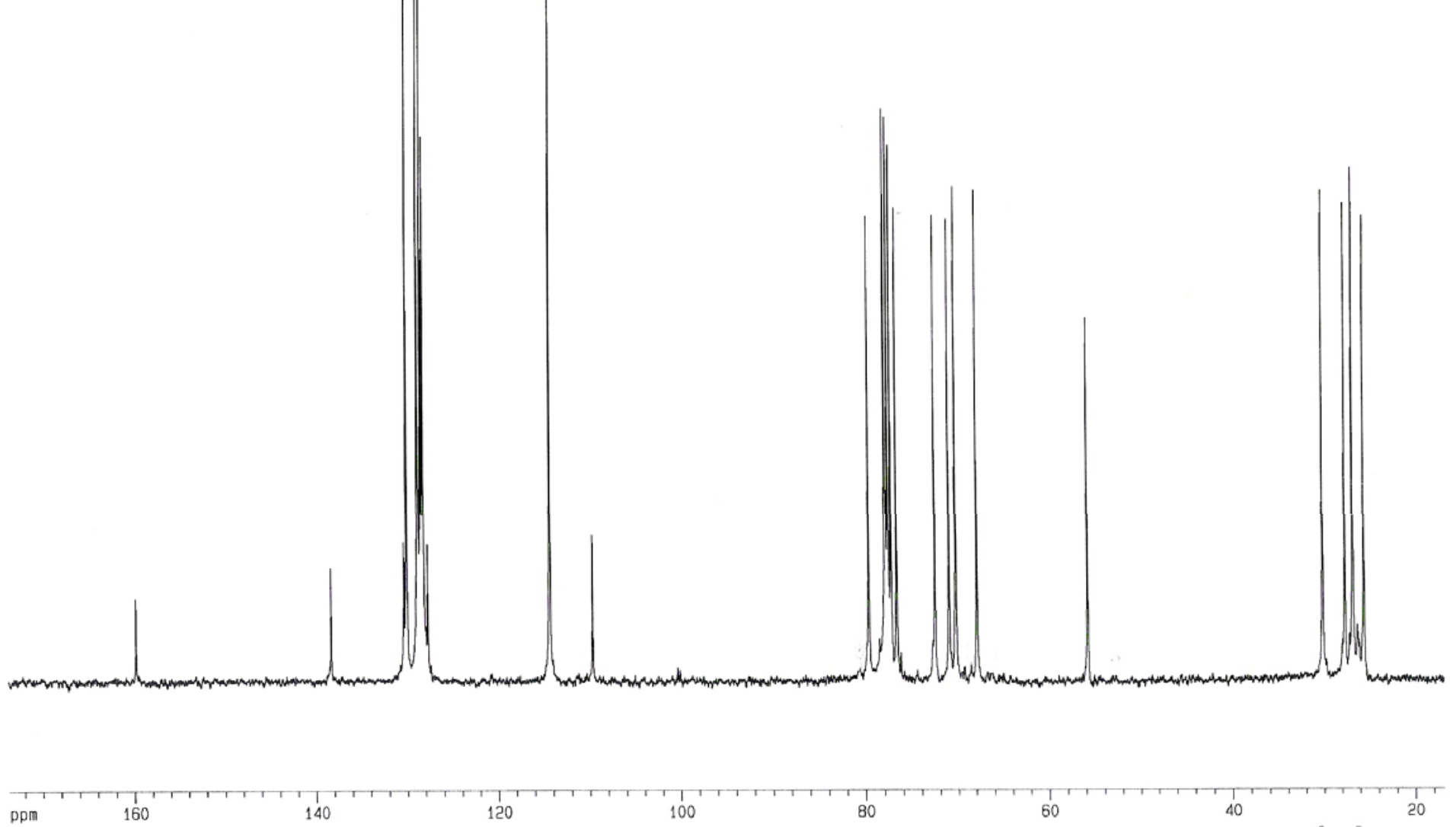


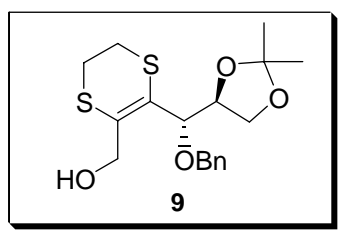

$75 \mathrm{MHz}^{\mathrm{CDCl}_{3}}$

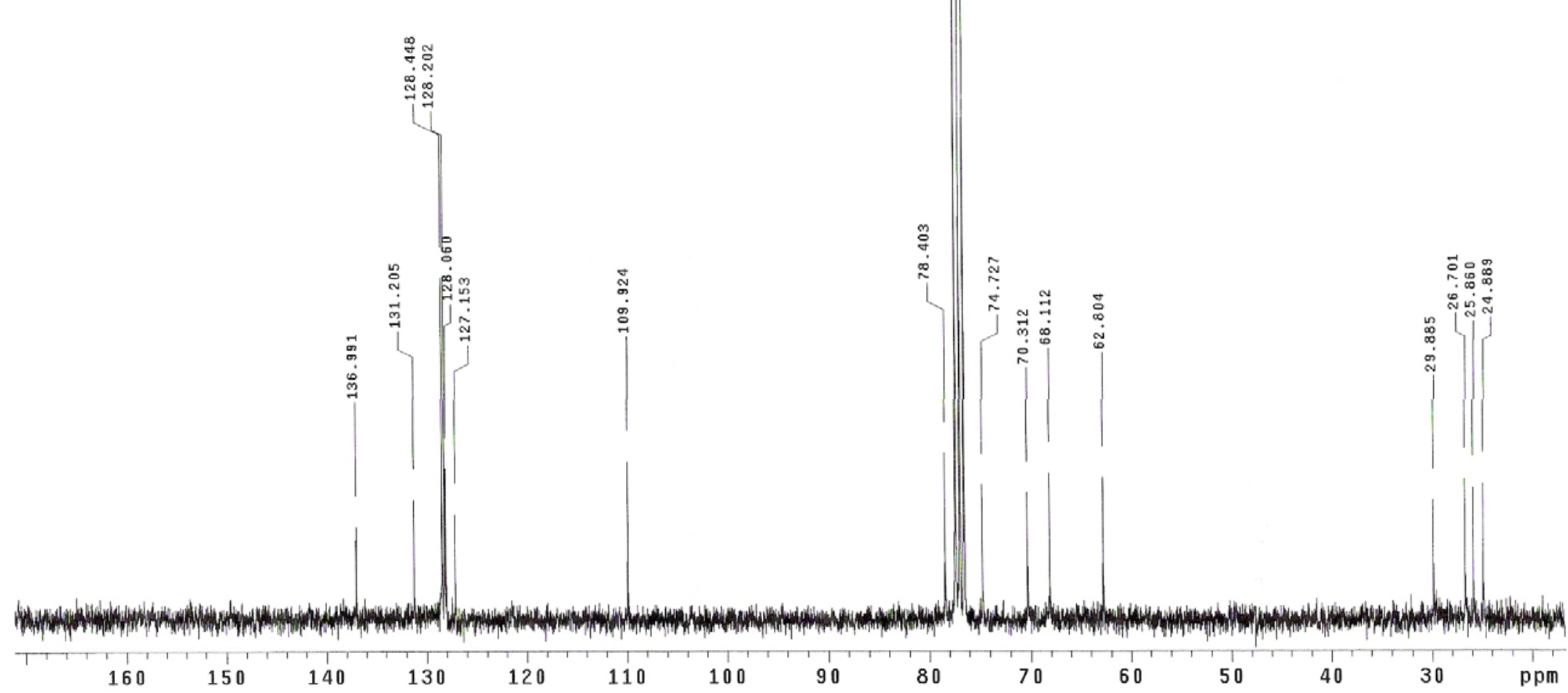




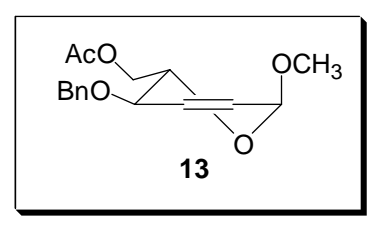

$100 \mathrm{MHz}, \mathrm{CDCl}_{3}$
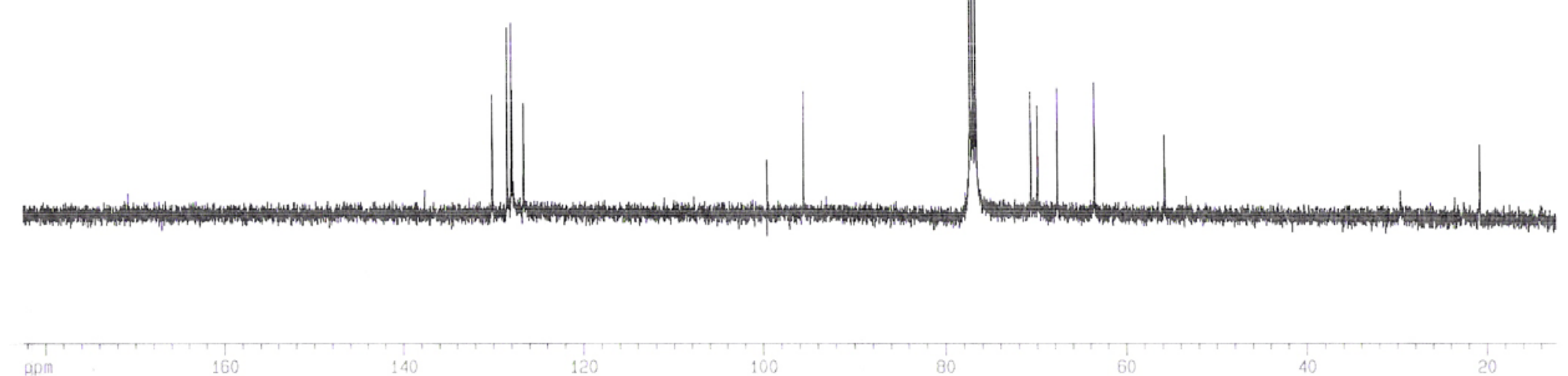


(14

$100 \mathrm{MHz}, \mathrm{CDCl}_{3}$

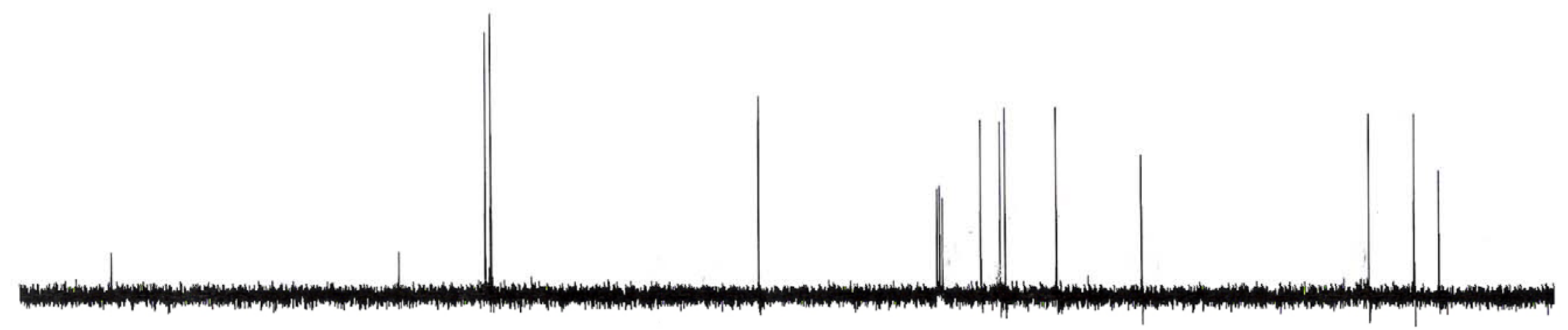

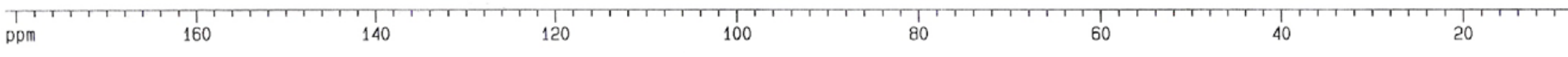



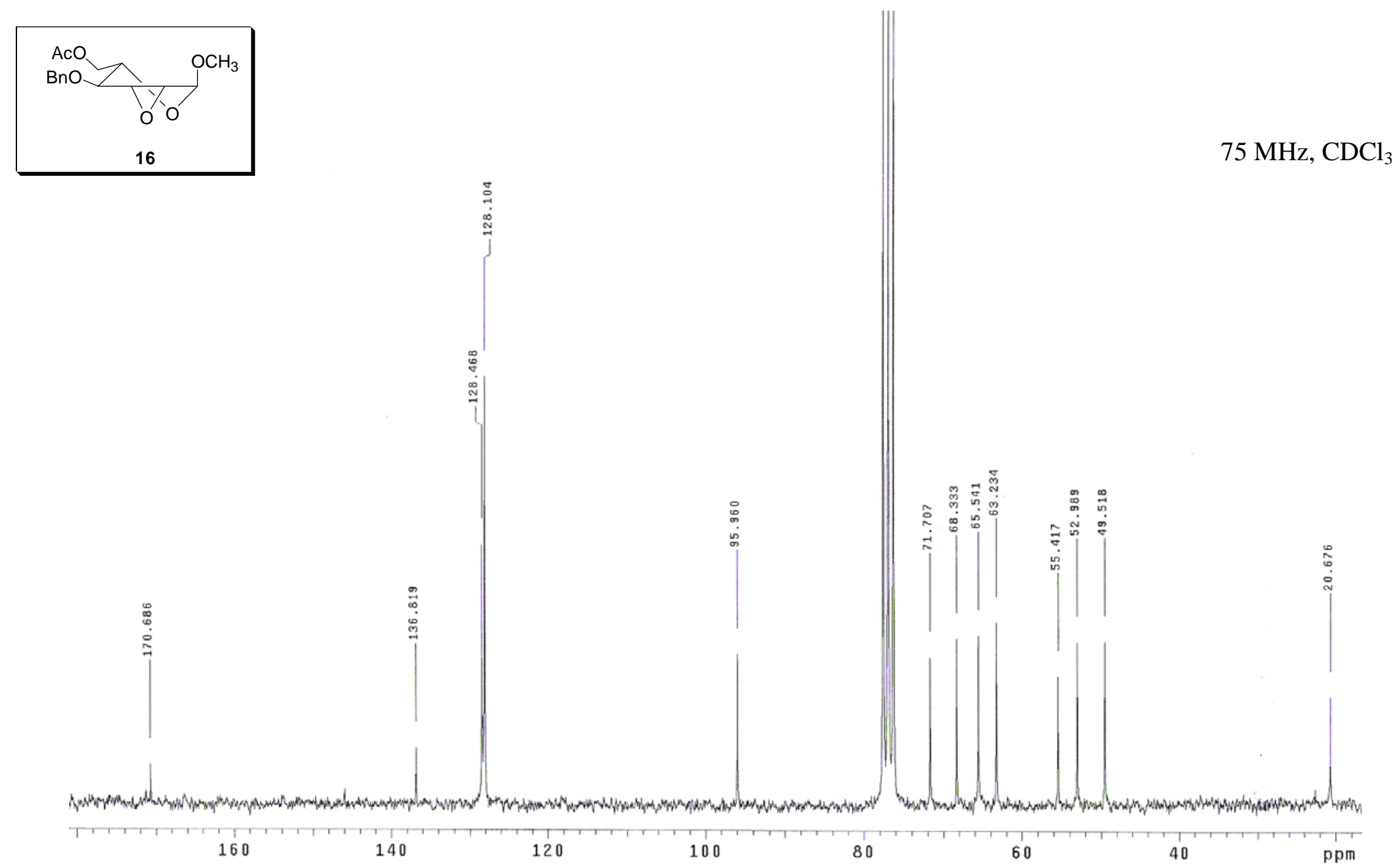


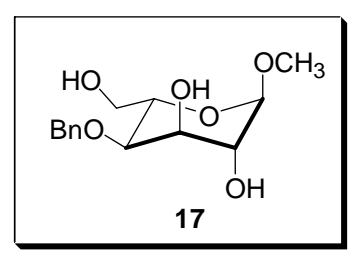

$125 \mathrm{MHz}, \mathrm{CDCl}_{3}$

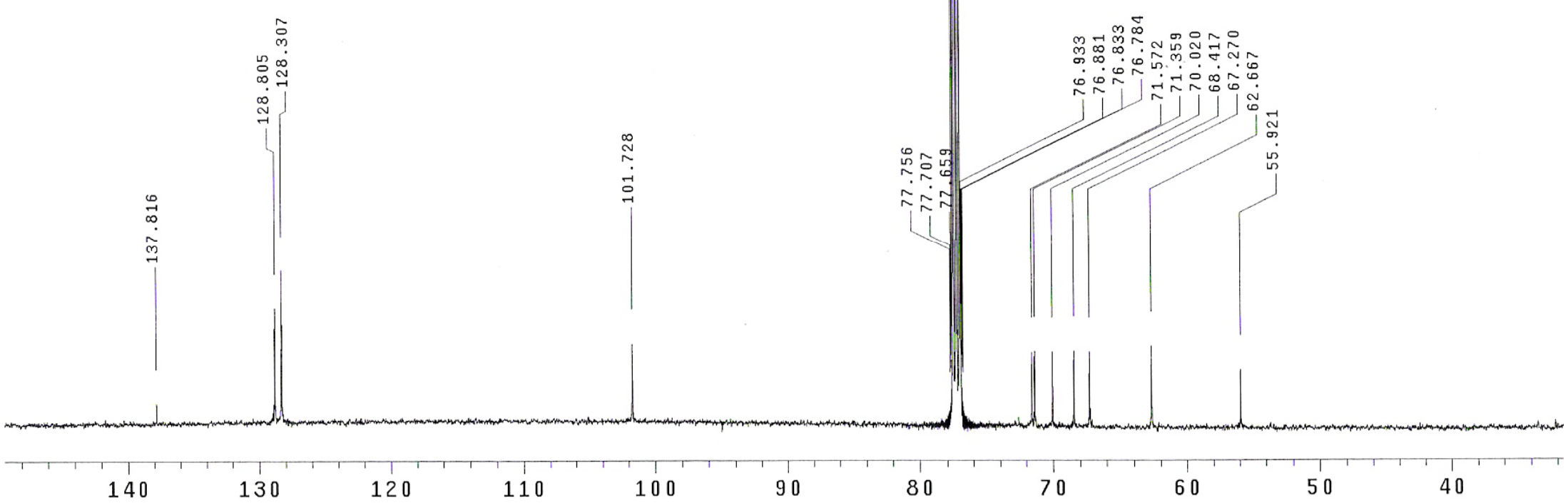




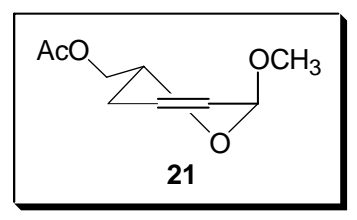

$125 \mathrm{MHz}^{\mathrm{CDCl}_{3}}$

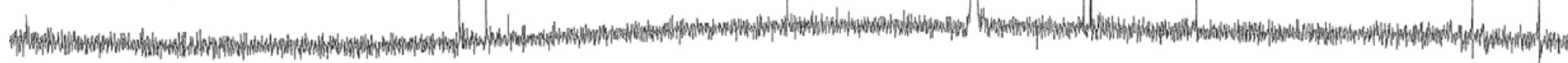

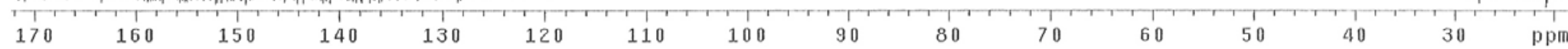




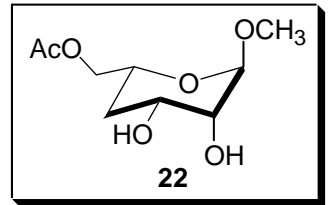

$125 \mathrm{MHz}, \mathrm{CDCl}_{3}$

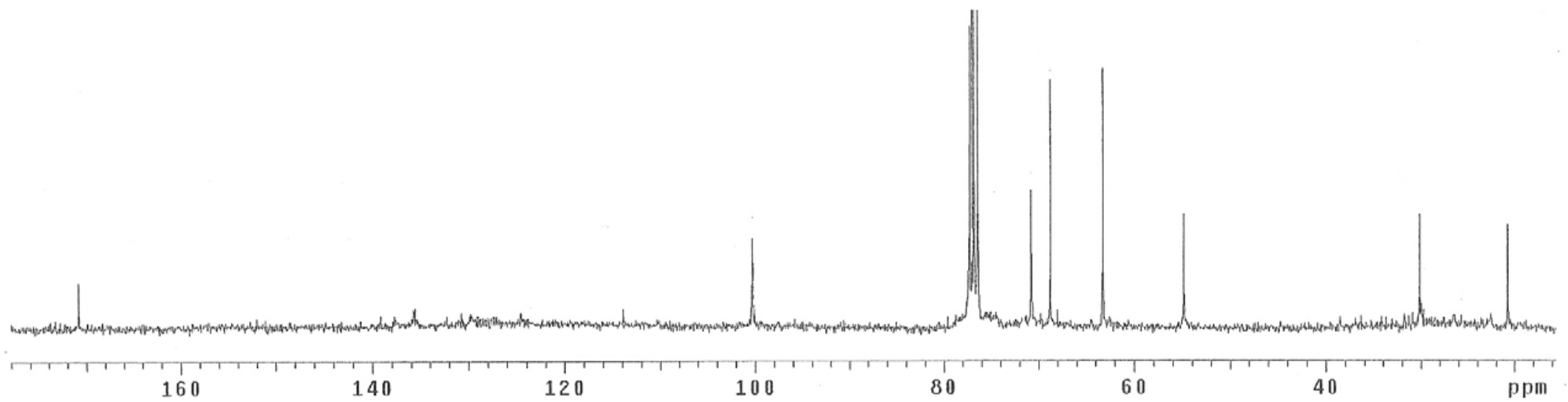




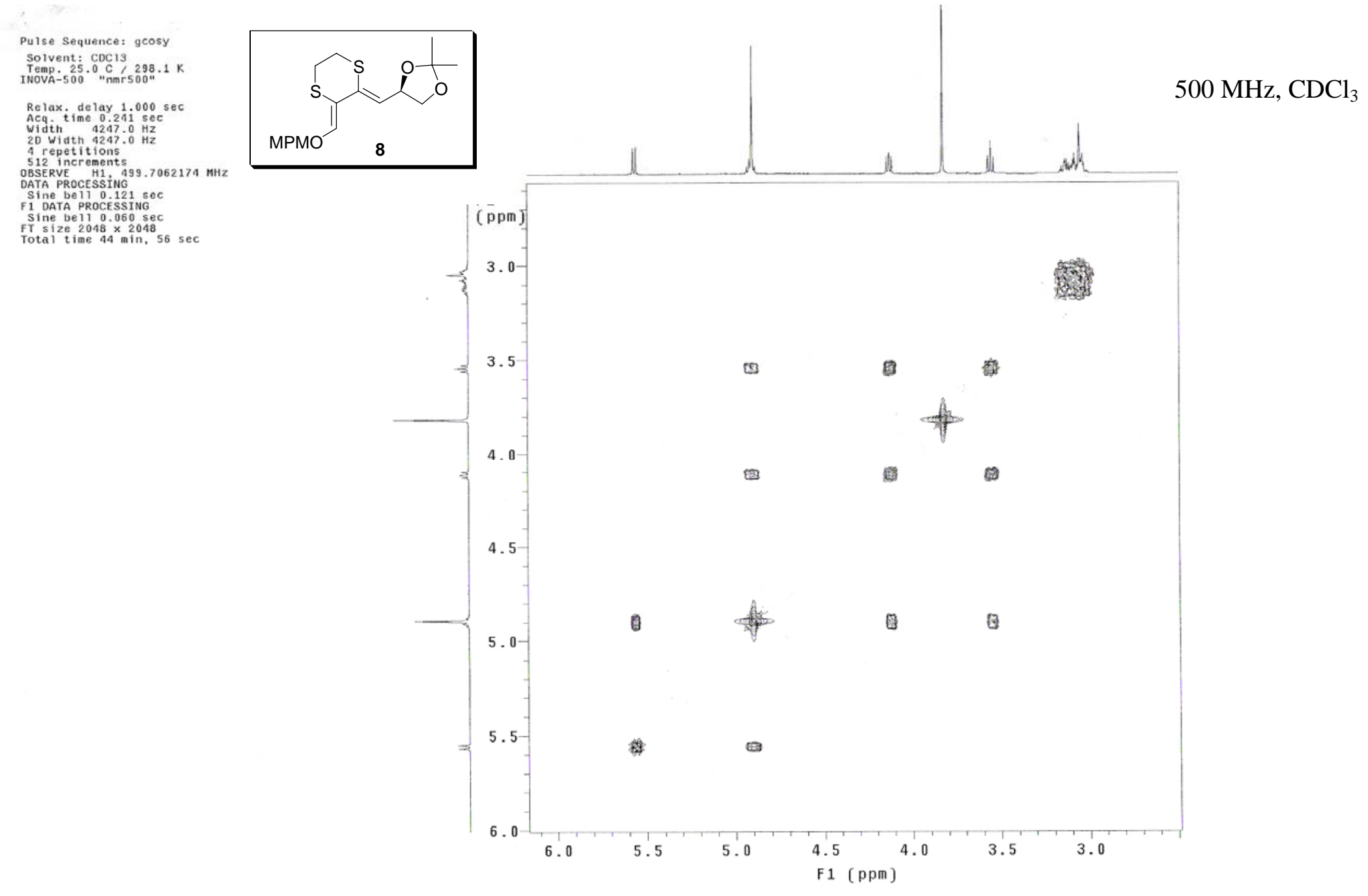


$500 \mathrm{MHz}, \mathrm{CDCl}_{3}$

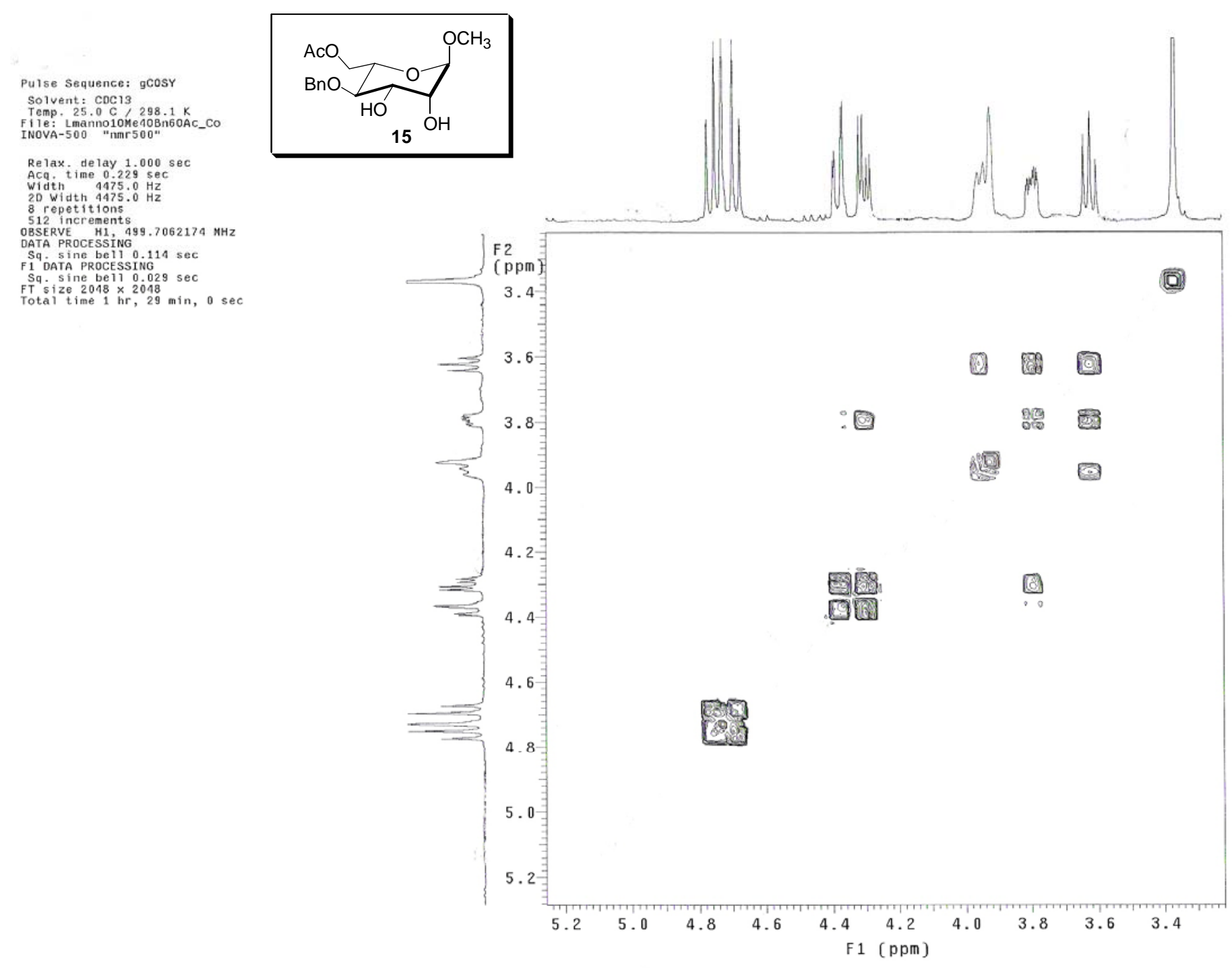


$500 \mathrm{MHz}, \mathrm{CDCl}_{3}$

STANDARD PROTON PARAMETERS

Pulse Sequence: gcosy

Solvent: $C^{2} C_{13} 13$ File: Altrosioco
INOVA-500 "nmis $500 "$

Re lax de lay $1.000 \mathrm{sec}$
Acq.

Wid 10 in $474.4 \mathrm{~Hz}$

8 repetitions

DBSERVE H1, 499.7062174 MHZ

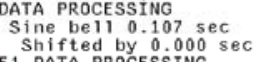

Sine be $110.054 \mathrm{sec}$

FT size $2008 \times 2048$
Total time $1 \mathrm{hr}, 27 \mathrm{~min}, 33 \mathrm{sec}$

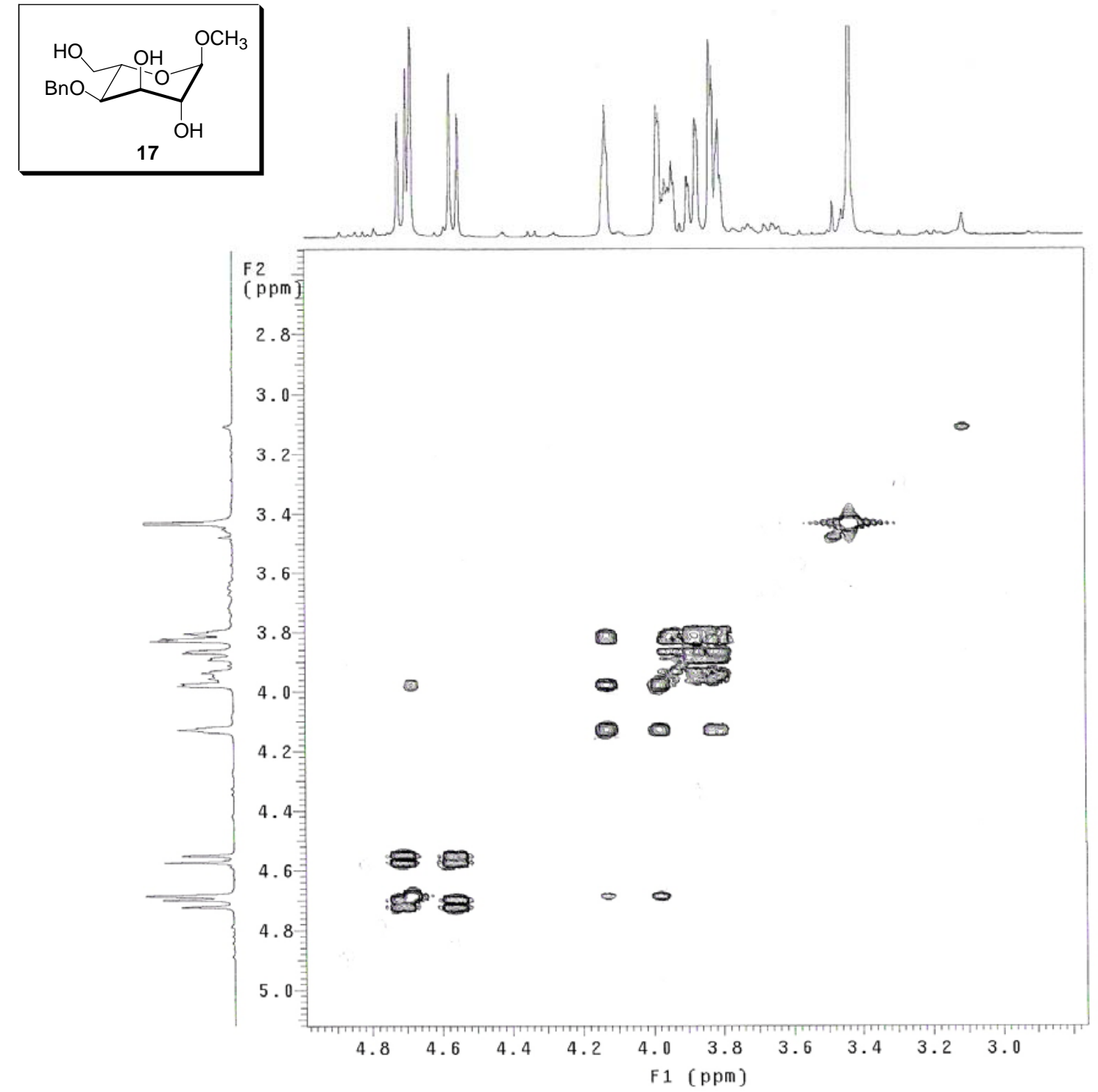

